\title{
Mechanisms for Maintaining Eukaryotic Replisome Progression in the Presence of DNA Damage
}

\author{
Thomas A. Guilliam * \\ Division of Protein and Nucleic Acid Chemistry, Medical Research Council Laboratory of Molecular Biology, Cambridge, \\ United Kingdom
}

The eukaryotic replisome coordinates template unwinding and nascent-strand synthesis to drive DNA replication fork progression and complete efficient genome duplication. During its advancement along the parental template, each replisome may encounter an array of obstacles including damaged and structured DNA that impede its progression and threaten genome stability. A number of mechanisms exist to permit replisomes to overcome such obstacles, maintain their progression, and prevent fork collapse. A combination of recent advances in structural, biochemical, and single-molecule approaches have illuminated the architecture of the replisome during unperturbed replication, rationalised the impact of impediments to fork progression, and enhanced our understanding of DNA damage tolerance mechanisms and their regulation. This review focusses on these studies to provide an updated overview of the mechanisms that support replisomes to maintain their progression on an imperfect template.

Nicholas Edward Dixon, University of Wollongong, Australia Rodrigo Reyes, McGill University, Canada Michael Trakselis, Baylor University, United States Aga Gambus, University of Birmingham, United Kingdom

*Correspondence:

Thomas A. Guilliam

guilliam@mrc-Imb.cam.ac.uk

Specialty section:

This article was submitted to

Structural Biology,

a section of the journal

Frontiers in Molecular Biosciences

Received: 21 May 2021 Accepted: 25 June 2021

Published: 06 July 2021

Citation:

Guilliam TA (2021) Mechanisms for Maintaining Eukaryotic Replisome

Progression in the Presence of

DNA Damage.

Front. Mol. Biosci. 8:712971. doi: 10.3389/fmolb.2021.712971

Keywords: DNA replication, replisome, DNA damage tolerance, translesion synthesis, repriming, recoupling, replication fork

\section{INTRODUCTION}

In eukaryotes, DNA replication is initiated from multiple origins across the genome (Robinson and Bell, 2005). Here, MCM double hexamers-loaded onto duplex DNA in G1 phase of the cell cycle-are activated upon entry into $S$ phase in a highly regulated manner, facilitated by the recruitment of numerous "firing factors" (Douglas et al., 2018). Many other origins remain dormant and only fire in response to replication impediments (Courtot et al., 2018). During activation, the lagging strand is ejected from each MCM ring to initiate unwinding of the parental duplex (Langston et al., 2017; Douglas et al., 2018), culminating in the formation of two active CMG (Cdc45-MCMGINS) holo-helicases encircling their respective leading-strand templates (Lewis and Costa, 2020). Each CMG helicase translocates $3^{\prime}-5^{\prime}$ on the leading strand to catalyse bidirectional fork propagation and additionally acts as a hub to coordinate the replicative enzymes and binding partners that collectively compose the replisome (Gambus et al., 2006; Fu et al., 2011; Pellegrini and Costa, 2016; Georgescu et al., 2017). Unwinding of the parental duplex provides a template for synthesis of nascent strands by the replicative polymerases (Pols) $\alpha, \delta$, and $\varepsilon$ (Guilliam and Yeeles, 2020a). Since the DNA double helix is antiparallel and all DNA polymerases catalyse synthesis in the $5^{\prime}-3^{\prime}$ direction, the nascent leading strand is synthesised mostly continuously in the same direction as replisome progression. Conversely, the lagging strand is replicated discontinuously in the opposite direction through repeated priming and extension to form Okazaki fragments. These are subsequently ligated together to generate a continuous daughter-strand molecule (KainumaKuroda and Okazaki, 1975; Burgers and Kunkel, 2017). 


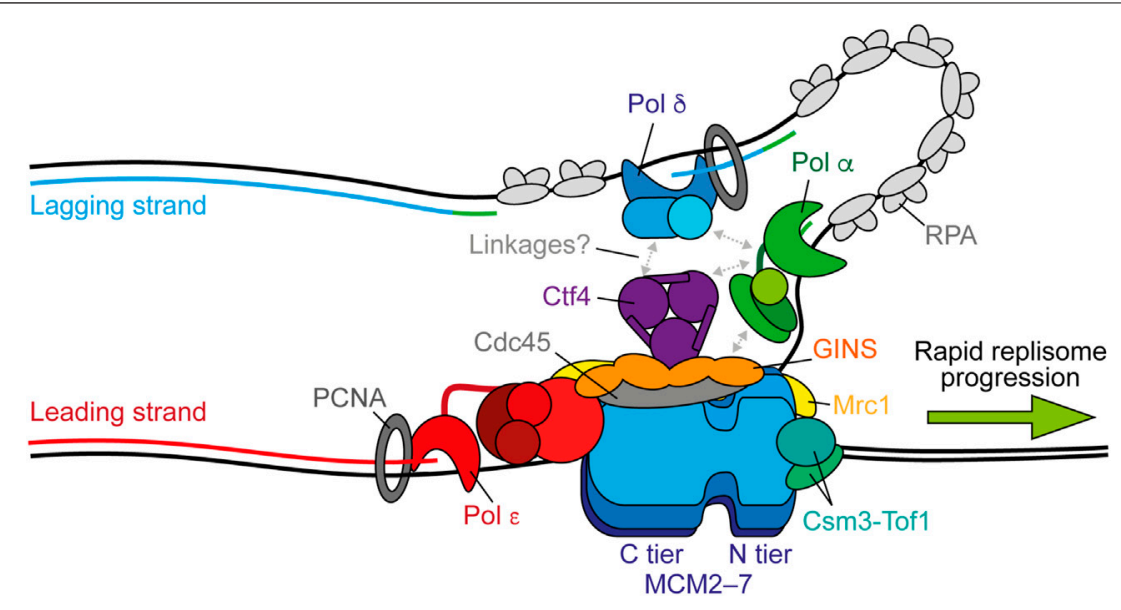

FIGURE 1 | Replisome architecture during unperturbed progression. CMG (Cdc45-GINS-MCM2-7) tracks along the leading strand to unwind the parental duplex. Pol $\varepsilon$ synthesises the leading strand continuously in complex with CMG. Pol $\alpha$ initiates discontinuous lagging-strand synthesis through regular priming. Primers are extended by Pol $\delta$ to form Okazaki fragments that are ligated together to produce a continuous daughter strand. Possible physical links between Pol $\alpha$, Pol $\delta$, Ctf 4 (And1), and CMG are indicated by gray arrows. Both Pol $\varepsilon$ and Pol $\delta$ associate with PCNA during synthesis and RPA binds any exposed ssDNA. Csm3-Tof1(TipinTimeless) bind ahead of CMG and contact the parental duplex. Mrc1 (Claspin) may bind across one side of CMG spanning the N and C tiers. Synthesis by Pol $\varepsilon$, Pol $\delta$, and $\mathrm{Pol} \alpha$ is shown in red, blue, and green respectively.

To efficiently complete DNA replication, yeast replisomes must traverse inter-origin distances up to 100 kilobases $(\mathrm{kb})$ before fork convergence and termination (Sekedat et al., 2010; Dewar and Walter, 2017). To cover such distances within S phase, replisomes progress at an average rate of $1.5-2 \mathrm{~kb} \mathrm{~min}^{-1}$ (Conti et al., 2007; Sekedat et al., 2010; Yeeles et al., 2017). However, genotoxic stress including DNA damage, DNA secondary structures and repeat sequences, interstrand and DNA-protein crosslinks, and limiting nucleotide levels can slow or stall replisome progression by inhibiting template unwinding and/or DNA synthesis (Zeman and Cimprich, 2014). Since CMG encircles and tracks along the leading strand, bulky obstacles located only in the lagging-strand template are more readily bypassed by the replisome ( $\mathrm{Fu}$ et al., 2011). Similarly, during lagging-strand replication, polymerase stalling at one Okazaki fragment does not affect synthesis of subsequent fragments or replisome progression, resulting only in the generation of a single-stranded (ss) DNA gap (Taylor and Yeeles, 2018). Here, DNA damage tolerance (DDT) occurs in a postreplicative gap-filling manner to complete synthesis of the daughter strand molecule. In contrast, bulky leading-strand obstacles can block CMG translocation while cessation of leading-strand synthesis significantly slows the replisome (Fu et al., 2011; Taylor and Yeeles, 2018, 2019). This necessitates the coordination of DDT mechanisms with the replisome to maintain canonical replication fork progression in the presence of leading-strand template aberrations.

In recent years, structural, biochemical, and single-molecule approaches have begun to decipher the architecture and mechanism of unperturbed replisome progression, the effect of impediments on this, and the details of DDT mechanisms used to maintain progression. This review highlights these recent studies to provide an overview of DDT mechanisms which maintain or restart efficient CMG progression following slowing or stalling of the replisome.

\section{REPLISOME ARCHITECTURE AND UNPERTURBED PROGRESSION}

The driving force of replisome progression is the ATP-dependent DNA unwinding activity of MCM, a two-tiered hetero-hexameric ring of $\mathrm{Mcm} 2-7$ subunits. One tier is composed of aminoterminal domains $(\mathrm{N}$ tier) and the other of carboxy-terminal AAA + ATPase domains ( $\mathrm{C}$ tier), with ATP-binding sites located at the subunit interfaces, which serve as the motor to power DNA template unwinding (Bell and Botchan, 2013). Loading of Cdc45 and GINS - a hetero-tetramer of Psf1-3 and Sld5-on to MCM during initiation forms the CMG holo-helicase and stabilises the $\mathrm{N}$ tier (Figure 1) (Costa et al., 2011; Yuan et al., 2016). Following the reconstitution of origin-dependent eukaryotic DNA replication with purified yeast proteins (Yeeles et al., 2015), 2D single-particle electron microscopy (EM) studies of reconstituted CMG revealed that the helicase translocates with an $\mathrm{N}$ tier first orientation (Douglas et al., 2018). This is consistent with the orientation suggested by a previous 3D cryo-EM structure (Georgescu et al., 2017) and that observed in more recent studies (Goswami et al., 2018; Eickhoff et al., 2019; Yuan et al., 2020a; Baretić et al., 2020).

Helicase translocation results from the allosteric coupling of ATP hydrolysis to movement of DNA-binding elements in each subunit lining the centre of the ring (Enemark and Joshua-Tor, 2006). Structures of homo-hexameric helicases support a rotary translocation mechanism (Enemark and Joshua-Tor, 2006; Thomsen and Berger, 2009; Itsathitphaisarn et al., 2012; Gao et al., 2019; Meagher et al., 2019). Here, five-subunits form a right-handed staircase surrounding the DNA with the sixth 
disengaged. Sequential ATP binding, hydrolysis, and product release facilitate subunit movement to escort DNA between each subunit and through the staircase (reviewed in Enemark and Joshua-Tor, 2008). However, studies of CMG translocation are complicated by the observation that not all ATPase domains are equally important among the MCM subunits (Ilves et al., 2010; Eickhoff et al., 2019). Insight into this process has been offered by high resolution cryo-EM structures of Drosophila CMG in three translocation states during unwinding (Eickhoff et al., 2019). This study proposed an asymmetric hand-over-hand rotational mechanism of CMG translocation in which binding of Mcm5-Mcm3 to the ATPase staircase occurs as a block, promoting a double step. This could explain the functional asymmetry of ATPase domains in the MCM ring (Eickhoff et al., 2019). A recent cryo-EM structure of human CMG bound to fork DNA is consistent with a sequential rotary model of translocation, but the ATP-hydrolysis status of $\mathrm{Mcm} 3$ differs from the asymmetric translocation model proposed by Eickhoff et al. (2019). Further studies are necessary to resolve if these differences represent speciesspecific variation in the CMG translocation mechanism.

Regardless of the precise mechanistic details, the $\mathrm{C}$ tier motor ring pulls DNA through the $\mathrm{N}$ tier to facilitate strand separation and replisome translocation. In contrast to classic strandexclusion models, where one strand is sterically excluded from the central channel of the helicase while the other is pulled through, duplex DNA enters the $\mathrm{N}$ tier of yeast CMG a short distance (Georgescu et al., 2017) and, moreover, the central channel is wide enough to completely accommodate duplex DNA (Wasserman et al., 2019). The $\mathrm{N}$ tier is subdivided into a zinc finger (ZF) ring at the front of CMG followed by an oligonucleotide-binding (OB) fold ring. A recent structure of yeast $\mathrm{CMG}$ revealed that separation of the parental duplex occurs at the bottom of the ZF sub-ring (Yuan et al., 2020a). Here, the lagging strand is diverted by the $\mathrm{OB}$ hairpin loops of $\mathrm{Mcm} 3$, $\mathrm{Mcm} 4, \mathrm{Mcm} 6$, and $\mathrm{Mcm}$ 7, with a possible exit channel between the ZF domains of $\mathrm{Mcm} 3$ and $\mathrm{Mcm}$ 5. This mechanism, referred to as the dam-and-diversion model, differs from the separation pin model, whereby a specific structural element separates the two strands. However, a putative strand-separation pin has been identified in a subsequent structure (Baretić et al., 2020). This is provided by a conserved phenylalanine in the $\mathrm{N}$-terminal hairpin loop of $\mathrm{Mcm} 7$ that abuts the final base pair before strand separation (Baretic et al., 2020). Whether this conserved residue is required for efficient unwinding remains to be determined. In both structures, the path of the DNA template is the same and therefore separation may be achieved by a combination of the two mechanisms.

Although activated CMG helicases reconstituted from an origin in vitro are sufficient for robust DNA synthesis in the presence of Pol $\alpha$ and $\operatorname{Pol} \varepsilon$, replication fork speeds are much slower than those observed in vivo in the absence of critical accessory proteins (Yeeles et al., 2015, 2017). These include the "fork protection complex" (FPC) comprised of Mrc1 (Claspin) and Csm3-Tof1 (Tipin-Timeless). A recent high resolution cryoEM structure of the FPC and CMG on forked DNA revealed that Csm3-Tof1 bind to MCM via Tof1 at the front of the replisome
(Figure 1) and grip the parental DNA duplex ahead (Baretić et al., 2020). Fork rate enhancement by Csm3-Tof1 is dependent on Mrc1 (Yeeles et al., 2017) which appears to bind across one side of CMG, spanning the $\mathrm{N}$ and $\mathrm{C}$ tiers (Baretić et al., 2020). The mechanism by which Mrc1 stimulates fork rates is not currently clear, however it also interacts with the flexible catalytic domain of Pol $\varepsilon$ (Lou et al., 2008), potentially aiding its correct positioning behind CMG (Zhou et al., 2017). Pol $\varepsilon$-comprised of the catalytic subunit Pol2 and three non-catalytic subunits Dpb2, Dpb3, and Dpb4-is a core component of the replisome required for origin firing (Sengupta et al., 2013; On et al., 2014). It binds stably to CMG via interactions between the noncatalytic C-terminal domain of Pol2 with $\mathrm{Mcm} 2$ and $\mathrm{Mcm} 5$ (Goswami et al., 2018) and, additionally, between Dpb2-the accessory subunit of Pol $\varepsilon$-with $\mathrm{Mcm} 3$ and the GINS subunit Psf1 (Sengupta et al., 2013; Sun et al., 2015; Goswami et al., 2018; Yuan et al., 2020b). Dpb2 may also serve to direct the leading strand from CMG to the Pol $\varepsilon$ active site (Yuan et al., 2020b). These interactions place Pol $\varepsilon$ in complex with and behind CMG to couple leading-strand synthesis to template unwinding (Figure 1) (Guilliam and Yeeles, 2020a), which is essential for maximum fork rates (Yeeles et al., 2017; Taylor and Yeeles, 2018, 2019). The homo-trimeric eukaryotic sliding clamp processivity factor, PCNA, additionally helps tether the flexible catalytic domain of $\mathrm{Pol} 2$ to the nascent leading strand, further contributing to maximum replisome progression rates in vitro (Yeeles et al., 2017).

Unlike leading-strand synthesis, which is mostly continuous, lagging-strand replication requires repeated priming by $\operatorname{Pol} \alpha$ and extension by Pol $\delta$ to generate Okazaki fragments (Figure 1) (Guilliam and Yeeles, 2020a). How Pol $\alpha$ is recruited to CMG to initiate primer synthesis is not currently understood. One potential mechanism is through Ctf4 (And-1), a trimeric hub that binds to CMG at the Cdc45-GINS interface (Yuan et al., 2019; Baretić et al., 2020; Rzechorzek et al., 2020) and interacts with a range of proteins including Pol $\alpha$ (Gambus et al., 2009; Simon et al., 2014; Villa et al., 2016; Guan et al., 2017; Kilkenny et al., 2017). However, Ctf4 is not required for DNA replication in vitro (Yeeles et al., 2015, 2017; Kurat et al., 2017) and only minimally affects retention of Pol $\alpha$ at the replisome in yeast cells (Kapadia et al., 2020). Instead, the Ctf4-Pol $\alpha$ interaction may be more important for recycling of parental histones on to the lagging strand, than for primer synthesis (Evrin et al., 2018; Gan et al., 2018). Curiously, a recent cryo-EM report demonstrated that $\mathrm{Ctf} 4$ can link two CMGs into a single "replication factory" (Yuan et al., 2019). However, further studies are required to confirm whether this occurs during active replication.

Until recently, Pol $\delta$ was assumed to function disconnected from the replisome, synthesising each Okazaki fragment while bound to PCNA before dissociating from DNA to permit ligation (Bell and Labib, 2016; Lancey et al., 2020a). However, two singlemolecule studies, one in vitro (Lewis et al., 2020) and one in vivo (Kapadia et al., 2020), have challenged this assumption by demonstrating that $\operatorname{Pol} \delta$ is retained at the replisome for multiple rounds of Okazaki fragment synthesis. It was suggested that Pol $\delta$ may interact with the replisome via Pol $\alpha$ 
(Huang et al., 1999; Johansson et al., 2004; Lewis et al., 2020). However, in vivo the residency time of Pol $\delta$ at the replisome exceeded that of Pol $\alpha$, indicating that it interacts with CMG independently, potentially through Ctf4 (Figure 1) (Bermudez et al., 2010; Kapadia et al., 2020). Since lagging-strand synthesis occurs in the opposite direction to CMG progression, these findings additionally posit the existence of lagging-strand loops (Figure 1). Unlike Pol $\varepsilon$, Pol $\delta$ is not required for maximum rates of replisome progression, but can support leading-strand synthesis, albeit more slowly, in the absence of active Pol $\varepsilon$ and is essential for complete lagging-strand synthesis (Yeeles et al., 2017). Consequently, although emerging evidence supports Pol $\delta$ as a stable component of the replisome, coupling of Pol $\delta$ to CMG does not appear to be important for maximum fork rates, likely because lagging-strand synthesis occurs in the opposite direction to replisome translocation. However, recent biochemical and cellular studies revealed that $\operatorname{Pol} \delta$ also plays a key role in the initiation of leading-strand synthesis. Here, it extends the first lagging-strand primer, generated by the oppositely translocating replisome, back across the origin to couple synthesis to CMG-Pol $\varepsilon$ and establish rapid fork progression (Garbacz et al., 2018; Aria and Yeeles, 2019).

\section{UNCOUPLING OF LEADING-STRAND SYNTHESIS AND FORK SLOWING}

Since the central channel of $\mathrm{CMG}$ is wide enough to accommodate duplex DNA, many DNA lesions can readily pass through the helicase, stalling DNA synthesis instead of CMG translocation (Zeman and Cimprich, 2014). Damaged nucleobases, abasic sites, and more bulky adducts such as pyrimidine dimers can be accommodated in the central channel of CMG and therefore do not pose a steric block to unwinding even when present in the leading-strand template (Taylor and Yeeles, 2018, 2019; Guilliam and Yeeles, 2021). However, the replicative polymerases are typically considered to be intolerant to DNA lesions and mismatched $3^{\prime}$ termini (Sale et al., 2012). The general structure of replicative polymerases is likened to a right hand; the catalytic site sits in the palm domain while the finger and thumb domains grip the template and primer (Sale et al., 2012). The intolerance of replicative polymerases is in part due to "induced fit" conformational changes during catalysis, whereby the finger domain moves to switch the polymerase from an open to a closed conformation only when the incoming dNTP correctly pairs with the templating base (Johnson, 2008; Freudenthal et al., 2013; Yang and Gao, 2018). The recent structure of the Pol $\varepsilon$ holoenzyme revealed that the finger domain tilts $27^{\circ}$ upon DNA-dNTP binding, changing the enzyme from a gapped circle conformation to one that completely encircles DNA ready for catalysis (Yuan et al., 2020b). The compact catalytic site prevents the accommodation of bulky DNA adducts, while $3^{\prime}$ mismatches weaken DNA binding to the active site triggering relocation to the $3^{\prime}-5^{\prime}$ proofreading exonuclease domain (Reha-Krantz, 2010). These features greatly improve fidelity and processivity but also make the enzyme intolerant to lesions which pass through
CMG (Yang and Gao, 2018). Similarly, limiting nucleotide levels and repetitive DNA sequences can stall synthesis without directly stopping CMG translocation (Zeman and Cimprich, 2014; Devbhandari and Remus, 2020).

Stalling of Pol $\delta$ on the lagging strand does not affect ongoing replisome progression or the synthesis of subsequent Okazaki fragments because priming occurs continually downstream, with respect to helicase translocation direction (Figure 2A) (Taylor and Yeeles, 2018). This leaves a short ssDNA gap which can be filled in behind the fork by classic DDT mechanisms (Leung et al., 2019). Moreover, Pol $\delta$ is not required for maximum fork rates (Yeeles et al., 2017). In contrast, stalling of Pol $\varepsilon$ causes uncoupling of leading-strand synthesis from template unwinding, resulting in slow uncoupled fork progression (Figure 2B). Here, template unwinding and lagging-strand synthesis continue in the absence of leading-strand synthesis but at a much-reduced rate (Taylor and Yeeles, 2018, 2019). Uncoupled forks can progress multiple kilobases (Taylor and Yeeles, 2018, 2019), generating long stretches of RPA-coated ssDNA on the leading strand, as observed in UV-irradiated yeast cells (Lopes et al., 2006). It is likely that uncoupling occurs as a result of the dissociation of the flexibly tethered catalytic domain of CMG-bound Pol $\varepsilon$ from the $3^{\prime}$ end of the nascent leading strand and PCNA (Figure 2B). This has been proposed to also occur spontaneously in the absence of genotoxic stress to populate the leading strand with PCNA for nucleosome assembly and mismatch repair (Georgescu et al., 2017; Zhou et al., 2017).

If not rapidly resolved, the RPA-coated ssDNA accumulated from uncoupling can activate the DNA replication checkpoint by providing a platform for recruitment of Mec1-Ddc2 in yeast, or ATR-ATRIP in vertebrates (Byun et al., 2005; Pardo et al., 2017). This subsequently activates the checkpoint effector kinase Rad53 in budding yeast, or CHK1 in vertebrates, to elicit the cellular stress response. In reconstituted DNA replication experiments, Rad53 was recently shown to further slow uncoupled CMG progression (Devbhandari and Remus, 2020). This may help prevent RPA depletion when uncoupling occurs for an extended period of time (Toledo et al., 2013). This mechanism was shown to be important in response to hydroxyurea (HU) which inhibits ribonucleotide reductase (RNR), resulting in dNTP depletion (Gan et al., 2017). In rad53-1 mutant yeast cells, HU treatment caused asymmetric DNA synthesis whereby extension proceeded much further along the lagging strand than the leading strand as a result of uncoupling. This may be due to a requirement for higher dNTP levels for leading-strand synthesis by $\mathrm{Pol} \varepsilon$ than lagging-strand synthesis by Pol $\delta$. Uncoupling in rad53-1 cells was suppressed by elevated dNTP levels (Gan et al., 2017). Importantly, checkpoint kinases upregulate dNTP levels in response to replication stress (Yeeles et al., 2013). Rad53 may therefore be important for limiting uncoupling by both directly preventing excessive template unwinding by CMG and promoting recoupling by elevating dNTP levels. Recent biochemical data demonstrate that direct slowing of replication forks by Rad53 is at least in part mediated by Mrc1 (McClure and Diffley, 2021, Preprint). Although Mrc1 functions upstream of Rad53 following 

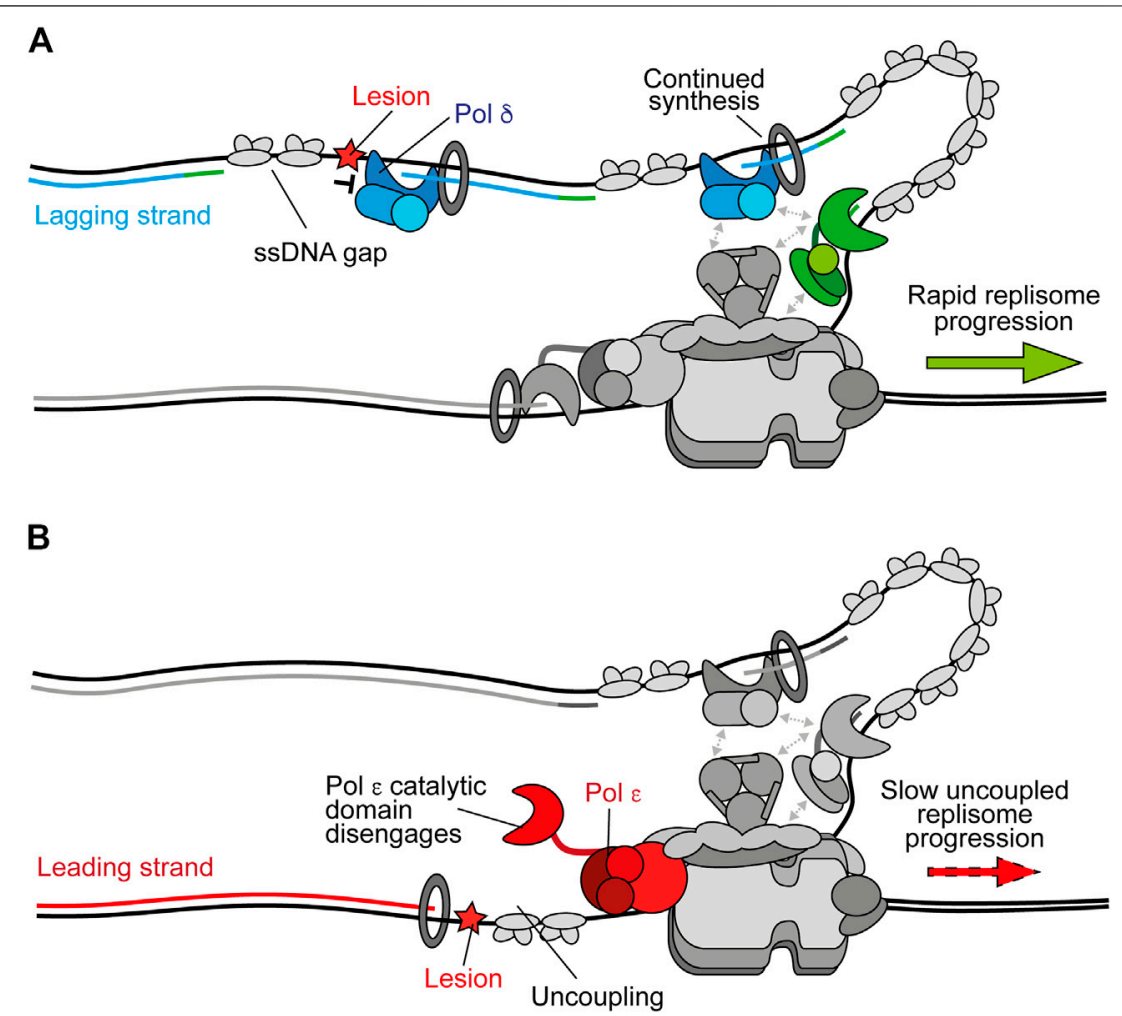

FIGURE 2 | Polymerase stalling on the leading and lagging strands. (A) Stalling of Pol $\delta$ at a DNA lesion during synthesis of one Okazaki fragment does not affect synthesis of subsequent fragments or ongoing replication fork speeds. Instead, a persistent ssDNA gap is formed. Synthesis by Pol $\delta$ and Pol $\alpha$ are shown in blue and green respectively. Other replisome components are shown in gray for clarity (B) Stalling of Pol $\varepsilon$ by a DNA lesion during leading-strand synthesis causes uncoupling due to disengagement of the flexible catalytic domain from the nascent strand and PCNA. Here, template unwinding and lagging-strand synthesis continue but at a much-reduced rate, resulting in the generation of RPA-coated SsDNA on the leading strand. Synthesis by Pol $\varepsilon$ is shown in red.

replication stress, it is also a target for phosphorylation by Rad53 and Mecl (Alcasabas et al., 2001). Phosphorylation of Mrc1 prevents it from stimulating $\mathrm{CMG}$, thereby slowing template unwinding (McClure and Diffley, 2021, Preprint).

Interestingly, inhibition of RNR by $\mathrm{HU}$ also elevates reactive oxygen species (ROS) in human cells (Somyajit et al., 2017). This can cause replication fork slowing via a checkpoint-independent mechanism. Here, peroxiredoxin 2 (PRDX2) acts as a ROS sensor. At low ROS levels it binds to Timeless in an oligomeric state. However, elevated ROS levels disrupt oligomerised PRDX2, causing its dissociation and the displacement of Timeless from the replisome, consequently slowing fork progression (Somyajit et al., 2017).

\section{REPLICASE SWITCHING AND RECOUPLING}

Accumulating evidence suggests that, in addition to its role as the lagging-strand replicase, $\mathrm{Pol} \delta$ replicates the leading strand whenever synthesis is not coupled to CMG (Guilliam and Yeeles, 2020a). Consistent with this, Pol $\delta$ acts as a "first responder" to stalling of leading-strand synthesis by outcompeting other polymerases, including free $\mathrm{Pol} \varepsilon$, for the uncoupled nascent strand
(Figure 3A) (Guilliam and Yeeles, 2020b). Once recruited, Pol $\delta$ can readily recouple the leading strand to CMG-Pol $\varepsilon$ whenever uncoupling occurs spontaneously or due to factors which are more readily tolerated by Pol $\delta$ than Pol $\varepsilon$ (Figure 3B) (Guilliam and Yeeles, 2020b). Indeed, we recently showed that Pol $\delta$ can efficiently bypass the oxidative single base lesions, thymine glycol and 8 oxoguanine, to recouple uncoupled forks in vitro (Guilliam and Yeeles, 2021). Similarly, Pol $\delta$ promotes recoupling following stalling of leading-strand synthesis at hairpin-forming sequences (Casas-Delucchi et al., 2021, Preprint). In conjunction, studies of vertebrate cells support a role for Pol $\delta$ in bypassing some lesions at the replication fork (Hirota et al., 2015, 2016). This replicase switch mechanism allows the replisome to quickly resume rapid progression following uncoupling, limiting ssDNA exposure on the leading strand while maintaining synthesis by the high-fidelity replicative polymerases; in turn avoiding potentially mutagenic DDT mechanisms or checkpoint activation. Similarly, Pol $\delta$ can excise and correct errors made by $\mathrm{Pol} \varepsilon$, whereas $\mathrm{Pol} \varepsilon$ can only correct its own misincorporations (Flood et al., 2015; Bulock et al., 2020). This suggests that misincorporated nucleotides that are not removed by Pol $\varepsilon$ may cause uncoupling, facilitating a switch to Pol $\delta$ before proofreading of the error and recoupling of leading-strand synthesis. A recent cryo-EM structure of the yeast Pol $\delta$ holoenzyme revealed a high degree of flexibility between the Pol3 catalytic and Pol31-Pol32 

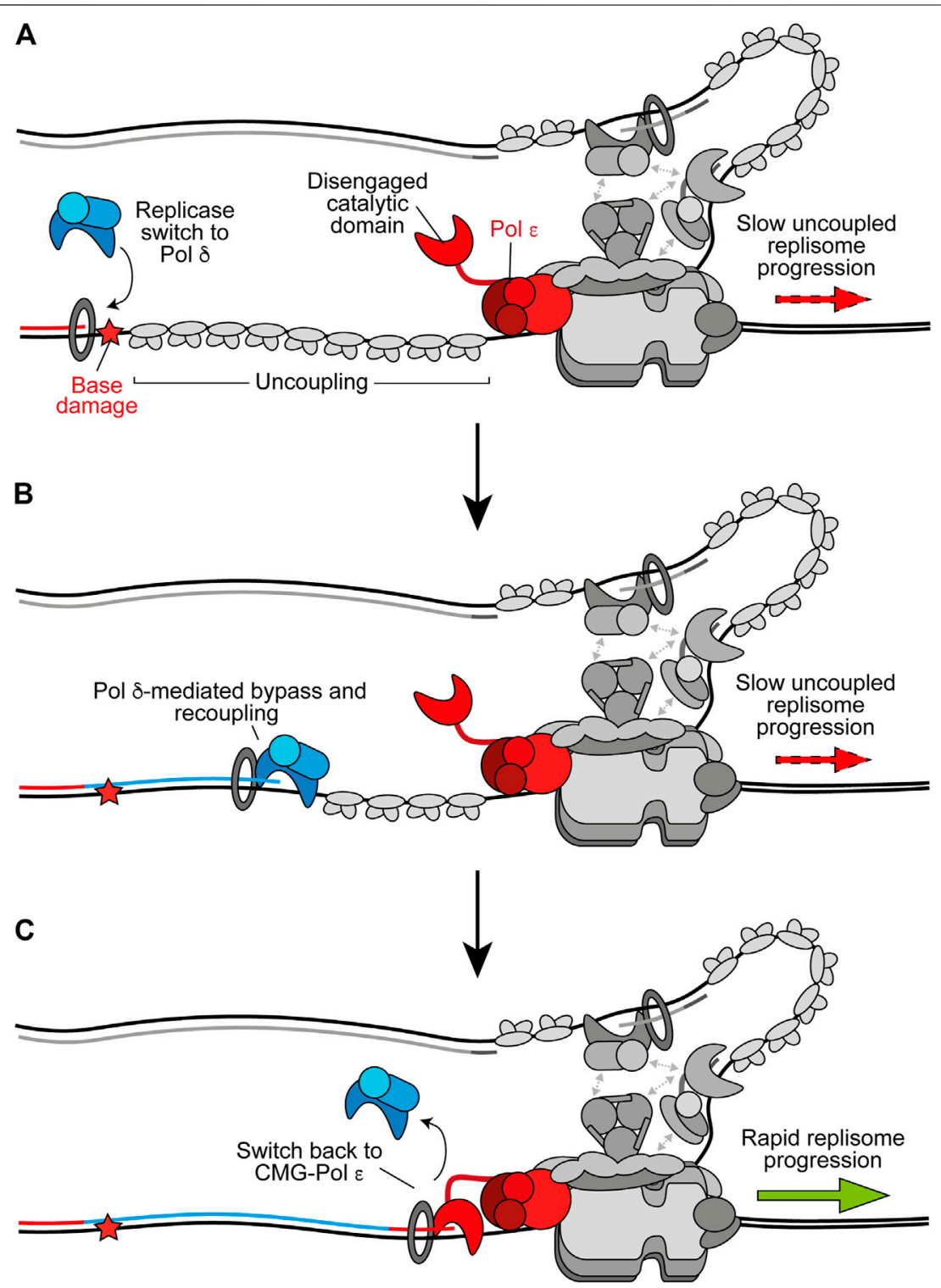

FIGURE 3 | Replicase switching and recoupling of leading-strand synthesis. (A) Upon uncoupling, a replicase switch occurs. Here, Pol $\delta$ is recruited to the uncoupled nascent leading strand. Other replisome components are shown in gray for clarity. (B) When stalling of Pol $\varepsilon$ occurs due to any factor that is more readily tolerated by Pol $\delta$, such as certain oxidative lesions, Pol $\delta$ directly extends the nascent leading strand through the impediment to recouple synthesis. (C) Upon recoupling the nascent leading strand is handed back to CMG-bound Pol $\varepsilon$ to restore rapid replisome progression. Synthesis by Pol $\varepsilon$ and Pol $\delta$ is shown in red and blue respectively.

regulatory subunits, which may aid proofreading and facilitate binding to a wider variety of DNA substrates (Jain et al., 2019).

Pol $\delta$ is likely favored for recoupling due to its faster rate of synthesis than the rate of uncoupled CMG progression, higher affinity than free Pol $\varepsilon$ for PCNA, and its ability to processively synthesise kilobases of DNA on an RPA-coated template in the presence of PCNA (Langston and O'Donnell, 2008; Georgescu et al., 2014; Yeeles et al., 2017; Sparks et al., 2019). Interestingly, a structure of Pol $\delta$-PCNA recently demonstrated that the polymerase holds the DNA substrate such that it is positioned through the centre of the PCNA clamp without direct PCNADNA contacts (Zheng et al., 2020). DNA-clamp interactions are instead mediated by water, allowing PCNA to function as a "water skate" to permit rapid and processive DNA synthesis while generating minimal resistance. Likewise, PCNA was recently shown to enhance nucleotide incorporation rates by Pol $\delta$ from 40 nucleotides per second to more than 350 per second (Mondol et al., 2019). This rate of nucleotide incorporation would allow the polymerase to rapidly catch up with uncoupled CMG, which has been estimated to unwind DNA at $~ 55$ base pairs per minute (Sparks et al., 2019; Devbhandari and Remus, 2020). It is not currently understood how the nascent leading strand is transferred from Pol $\delta$ back to CMG-Pol $\varepsilon$ (Figure 3C). However, unlike free Pol $\varepsilon$, CMG-Pol $\varepsilon$ does 


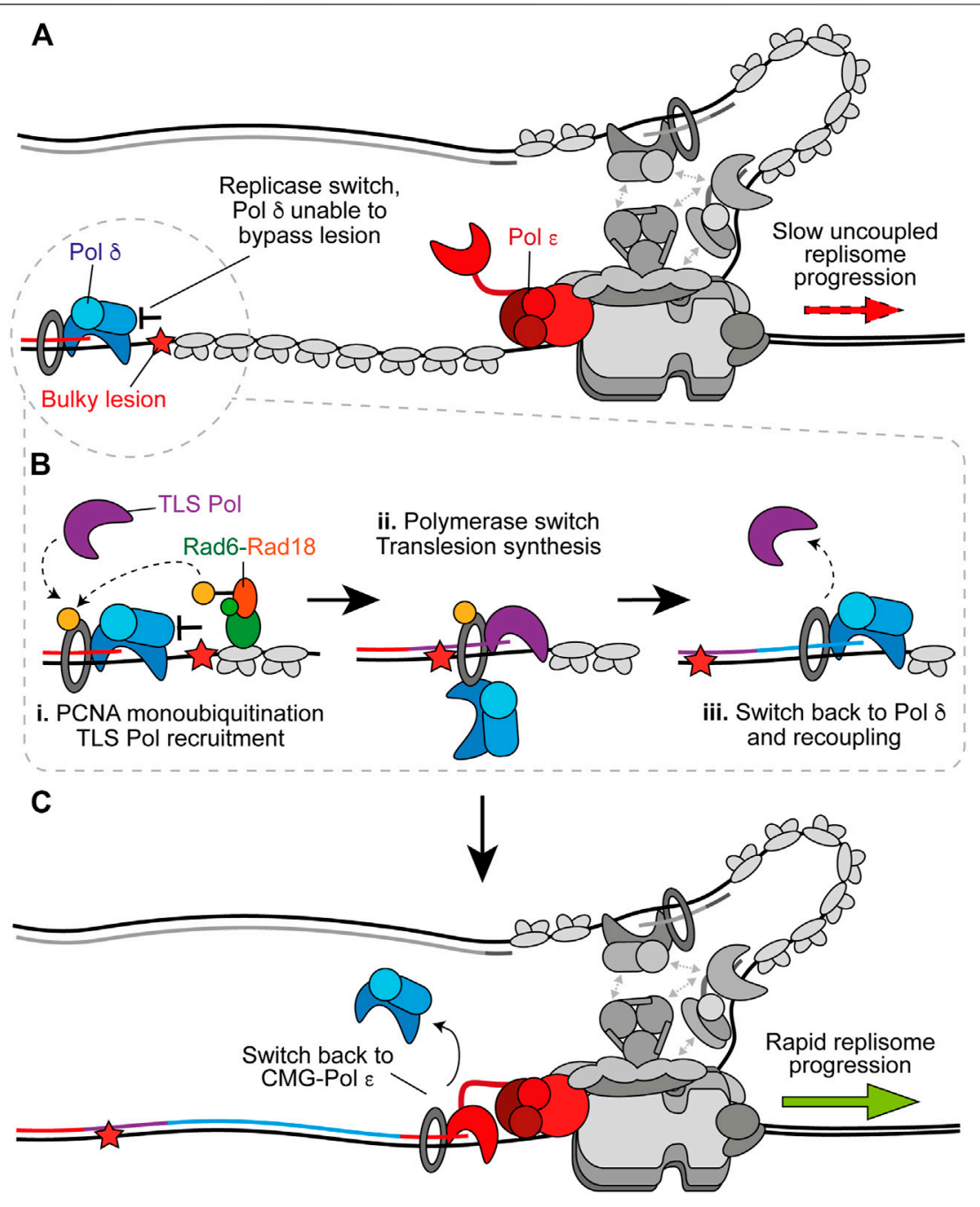

FIGURE 4 | On the fly translesion synthesis can promote recoupling. (A) When Pol $\delta$ is recruited to the leading strand following uncoupling, but is unable to bypass the impediment, TLS is required prior to recoupling. Other replisome components are shown in gray for clarity. (B) i. RPA-coated ssDNA recruits Rad6-Rad18 which catalyse monoubiquitination of PCNA. This stimulates recruitment of a TLS polymerase. ii. The TLS polymerase extends the nascent strand past the impediment. This could occur without complete dissociation of Pol $\delta$ from PCNA. iii. After bypass a switch back to Pol $\delta$ facilitates rapid recoupling. (C) Pol $\delta$ hands the nascent leading strand back to Pol $\varepsilon$ to restore canonical fork rates. Synthesis by Pol $\varepsilon$, Pol $\delta$, and the TLS polymerase is shown in red, blue, and purple respectively.

outcompete Pol $\delta$ for the leading strand (Georgescu et al., 2014). It is possible handover occurs through collision release in which Pol $\delta$ is ejected from PCNA upon running into uncoupled CMG, similar to the mechanism proposed to occur during Okazaki fragment synthesis (Langston and O'Donnell, 2008; Schauer and O'Donnell, 2017). Regardless, rapid fork rates are reinstated following Pol $\delta$-mediated recoupling, suggesting CMG-Pol $\varepsilon$ efficiently resumes leading-strand synthesis downstream (Guilliam and Yeeles, 2020b).

\section{TRANSLESION SYNTHESIS POLYMERASES}

Despite a greater ability to tolerate certain single-base lesions than Pol $\varepsilon$ (Guilliam and Yeeles, 2021), Pol $\delta$ also stalls upon encountering more bulky or distorting lesions such as a cyclobutane pyrimidine dimer (CPD), a well-characterised UV-induced photoproduct (Taylor and Yeeles, 2018; Guilliam and Yeeles, 2020b). In these instances, specialised translesion synthesis (TLS) polymerases are required for direct synthesis across damage prior to recoupling (Guilliam and Yeeles, 2020b). TLS that functions to promote recoupling is termed "on the fly" TLS (Figure 4) to distinguish it from gap-filling TLS. The main TLS polymerases in eukaryotes are those of the Y-family; Pol $\eta$, $\mathrm{Pol} \iota$, Pol $\kappa$, and Rev1, in addition to the B-family polymerase Pol $\zeta$ (Sale et al., 2012). Of these, only Pol $\eta$, Rev1, and Pol $\zeta$ are present in budding yeast. The Y-family polymerases have spacious solvent accessible active sites formed between the palm and finger domains that make few, mostly non-specific, contacts with the DNA template, permitting the accommodation of bulky lesions and template distortions (Fleck and Schär, 2004; 
Sale et al., 2012). They also possess an additional "little finger" domain, not present in other polymerase families, which together with the thumb domain grips duplex DNA and may play a role in lesion specificity (Boudsocq et al., 2004; Fleck and Schär, 2004; Wilson et al., 2013). Compared to the replicative polymerases, the finger domain of the Y-family polymerases is stubbier and remains in a closed conformation irrespective of DNA-dNTP binding, thereby removing the "induced fit" mechanism of dNTP selection (Vaisman and Woodgate, 2017). They also lack proofreading exonuclease activity and therefore the ability to excise mispaired nucleotides. Consequently, the Y-family polymerases gain an increased tolerance of template lesions at the expense of fidelity and processivity.

$\mathrm{Pol} \zeta$ is thought to function as an extender when two polymerases are required for lesion bypass. Here, a Y-family polymerase first incorporates nucleotides opposite the damage, before $\mathrm{Pol} \zeta$ takes over to catalyse extension beyond the lesion in a process that is typically error-prone (Johnson et al., 2000). Only recently was the structure of $\mathrm{Pol} \zeta$ determined (Malik et al., 2020), revealing that it adopts a pentameric ring conformation composed of the catalytic subunit Rev3, two Rev7 subunits, and the non-catalytic Pol31 and Pol32 subunits which are shared with Pol $\delta$ (Johnson et al., 2012; Makarova et al., 2012). Similar to the replicative polymerases, the finger domain transitions from an open to a closed conformation upon binding the correct $\mathrm{dNTP}$, conferring higher fidelity during nucleotide selection compared to the Y-family polymerases (Malik et al., 2020). However, key differences between Pol $\zeta$ and Pol $\delta$ explain the former's penchant for extending aberrantly paired $3^{\prime}$ ends (Johnson et al., 2000). In Pol $\delta$, the linker between the NTD and palm domain contacts the terminal base pair to prevent efficient extension of a mismatch. However, these contacts are not present in $\mathrm{Pol} \zeta$ and although the polymerase contains an exonuclease domain, it is inactive. These features make Pol $\zeta$ well suited to fulfill an extender role during TLS and reveal why, despite having higher fidelity than the Y-family polymerases (McCulloch and Kunkel, 2008), it is involved in almost all damage-induced mutagenesis (Lawrence, 2002).

\section{THE POLYMERASE SWITCH DURING TLS}

On the fly TLS can rescue uncoupled forks when Pol $\delta$ alone is unable to recouple the leading strand (Figure 4A) (Guilliam and Yeeles, 2020b). However, Pol $\delta$ outcompetes TLS polymerases for binding to stalled nascent $3^{\prime}$ ends and in doing so limits mutagenesis by negatively regulating TLS when it is not required for recoupling (Guilliam and Yeeles, 2020b). RPAcoated ssDNA, generated by persistent stalling, recruits the E2-E3 ubiquitin ligase complex, Rad6-Rad18 (Watanabe et al., 2004; Davies et al., 2008), which facilitates monoubiquitination of PCNA to promote a switch from Pol $\delta$ to TLS polymerase to facilitate lesion bypass (Figure 4B) (Hoege et al., 2002; Stelter and Ulrich, 2003; Bienko et al., 2005; Garg and Burgers, 2005; Plosky et al., 2006; Guilliam and Yeeles, 2020b). Y-family TLS polymerases are recruited to monoubiquitinated PCNA via their ubiquitin-binding ZF (Pol $\eta$ and Pol $\kappa$ ) or helical ubiquitin-binding motifs (Pol $\iota$ and Rev1), in addition to canonical PCNA interacting peptide (PIP) box motifs in their non-catalytic C-terminal extensions (Leung et al., 2019). Rev1 lacks a PIP box and instead interacts with PCNA via its N-terminal BRCT and little finger domains (Guo et al., 2006; Sharma et al., 2011).

The ubiquitin moiety is located on the back side of PCNA (Freudenthal et al., 2010). This may allow TLS polymerases to be recruited to the back face of monoubiquitinated PCNA while Pol $\delta$ remains bound at the front (Freudenthal et al., 2010). This toolbelt model, in which multiple binding partners can occupy PCNA monomers not bound by $\operatorname{Pol} \delta$, is supported by the recent structure of Pol $\delta$-PCNA-FEN1 in complex on DNA (Lancey et al., 2020a) which suggests a toolbelt mechanism for flap cleavage during Okazaki fragment maturation. This study also revealed that PCNA can tilt up to $20^{\circ}$, disrupting interactions between the catalytic subunit of Pol $\delta$ and PCNA that are critical for DNA synthesis, while the polymerase remains bound to PCNA via the PIP box located in its thumb domain (Lancey et al., 2020a). The same group recently solved the structure of Pol $\kappa$-PCNA-DNA and stalled Pol $\delta$-PCNA-DNA to propose a mechanism of polymerase switching (Lancey et al., 2020b, Preprint). Therein, Pol $\delta$ releases the primer-template from the active site upon stalling, Pol $\kappa$ binds to an exposed PCNA protomer in a flexible state and either actively displaces Pol $\delta$, or Pol $\delta$ independently dissociates, from DNA to form the final Pol $\kappa$ holoenzyme. Importantly, tilting of PCNA provides enough room to accommodate active Pol $\kappa$ and retain Pol $\delta$ on PCNA via its thumb domain PIP box. Pol $\delta$ might therefore hand over synthesis to the TLS polymerase without dissociating from PCNA to allow a rapid switch back after lesion bypass (Figure 4B), before subsequent recoupling (Figure 4C). Importantly, evidence supports a toolbelt model of polymerase switching in E. coli (Indiani et al., 2005; Kath et al., 2016) and archaea (Cranford et al., 2017), giving credence to the prospect that a similar mechanism occurs in eukaryotes. However, it is also possible that Pol $\delta$ fully dissociates from PCNA and DNA to permit TLS before reassociating to recouple synthesis.

In addition to PCNA, the C-terminal domain of Rev1 interacts with the other Y-family TLS polymerases through PIP-like motifs (Boehm and Washington, 2016), and binds the Rev7 subunit of Pol $\zeta$ (Murakumo et al., 2001; Zhao and Washington, 2017). Numerous studies support a non-catalytic role for Rev1 in the recruitment of other TLS polymerases (Nelson et al., 2000; Haracska et al., 2001; Ross et al., 2005; Edmunds et al., 2008). One proposed mechanism is through the formation of "Rev1 bridges" in which a TLS polymerase is linked to PCNA via Rev1 without interacting directly with the clamp. Both PCNA toolbelts and Rev1 bridges have been observed in single-molecule studies and they can interchange dynamically without dissociation (Boehm et al., 2016). The relative contribution of PCNA monoubiquitination and Rev1 in recruiting and coordinating TLS is likely to be lesion specific (Wang and Xiao, 2020). Indeed, accurate CPD bypass by $\mathrm{Pol} \eta$ does not require Rev1 or Pol $\zeta$ but is dependent on PCNA monoubiquitination (Kannouche et al., 2004; Bienko et al., 2005; Andersen et al., 
2011; Guilliam and Yeeles, 2020b), whereas BaP-dG ( $N^{2}$-benzo[a] pyrene-dG) bypass by $\mathrm{Pol} \kappa$ is dependent on its interaction with Rev1 (Ohashi et al., 2009). The requirement for Rev1 is more important for two-polymerase TLS that also requires $\mathrm{Pol} \zeta$ for extension and is typically error-prone (Livneh et al., 2010). Moreover, the affinity of different TLS polymerases for Rev1 varies, in some cases being relatively weak and allowing competition (Pustovalova et al., 2016). Consequently, Rev1 may help select a different polymerase if the first cannot bypass the lesion following recruitment via monoubiquitinated PCNA, switching TLS from a one to a two-polymerase mode where necessary. In addition to Rev7, Rev1 was more recently found to also interact with the PolD3 (Pol32 in yeast) subunit of human Pol $\zeta / \mathrm{Pol} \delta$ with higher affinity than it does with other Y-family polymerases (Pustovalova et al., 2016). This interaction may help displace the "inserter" polymerase from Rev1 and monoubiquitinated PCNA to facilitate extension by Pol $\zeta$ beyond the lesion (Pustovalova et al., 2016). The sharing of Pol31-Pol32 between Pol $\delta$ and Pol $\zeta$ led to the proposal that removal of Pol3 and recruitment of Rev3-Rev7 at the site of damage coordinates a switch between the two (Baranovskiy et al., 2012). In support, Pol3 is degraded in response to damage (Daraba et al., 2014). However, Pol $\zeta$ exists as a stable foursubunit complex (Rev3-Rev7-Pol31-Pol32) in all stages of the cell cycle irrespective of damage, potentially arguing against subunit exchange with Pol $\delta$ (Makarova et al., 2012).

\section{REPRIMING}

Aside from TLS, uncoupled replication forks can be rescued by reinitiating leading-strand synthesis through the generation of a new primer downstream of the impediment in a process termed repriming. Similar to ongoing Okazaki fragment synthesis after Pol $\delta$ stalling on the lagging strand, this leaves behind a ssDNA gap which can be filled by TLS or template switching. In reconstitution experiments with yeast proteins, Pol $\alpha$ could readily synthesise primers on the lagging strand but was inefficient at repriming the leading strand due to inhibition by RPA (Taylor and Yeeles, 2018). This disparity between leading and lagging-strand priming efficiency suggests these two processes are mechanistically distinct. It is likely that laggingstrand priming is facilitated by the position of Pol $\alpha$ in the replisome, while leading-strand repriming uses free Pol $\alpha$ and is therefore sensitive to RPA concentrations. Coupling of leadingstrand synthesis during initiation occurs via extension of a lagging-strand primer back across the origin by Pol $\delta$ (Garbacz et al., 2018; Aria and Yeeles, 2019), further arguing against an efficient Pol $\alpha$-mediated leading-strand priming mechanism in unperturbed conditions. However, it is possible that leading-strand repriming by $\mathrm{Pol} \alpha$ occurs under conditions of RPA exhaustion, or requires factors or modifications not present in the reconstituted system (Lopes et al., 2006; Fumasoni et al., 2015; Toledo et al., 2017; Taylor and Yeeles, 2018).

In higher eukaryotes, leading-strand repriming is catalysed by a second archaeo-eukaryotic primase named PrimPol that is absent in budding yeast (Bianchi et al., 2013; García-Gómez et al., 2013; Wan et al., 2013; Guilliam et al., 2015b; Guilliam and Doherty, 2017). PrimPol interacts directly with RPA and this interaction is required for recruitment of the primase to the ssDNA generated by uncoupling (Figure 5A) (Guilliam et al., 2015a, 2017; Šviković et al., 2019). The naming of PrimPol reflects its ability to perform both DNA primase and polymerase activities, and similar to the TLS polymerases it can directly bypass a number of lesions in vitro (Bianchi et al., 2013; Keen et al., 2014b). However, the catalytic domain of PrimPol does not resemble a canonical polymerase fold, completely lacks a thumb subdomain, makes almost no contacts with the primer strand, has very low inherent processivity, and does not noticeably interact with PCNA (Keen et al., 2014b; Guilliam et al., 2015a; Rechkoblit et al., 2016). Likewise, although PrimPol can bypass a (6-4) T-T photoproduct, its active site is not able to accommodate this lesion and it is likely bypassed by a looping out mechanism that generates deletions (Bianchi et al., 2013; Rechkoblit et al., 2016; Guilliam and Doherty, 2017). Moreover, the primase but not polymerase activity of PrimPol is dependent on its noncatalytic C-terminal ZF domain, which binds and selects the first $5^{\prime}$ nucleotide of the nascent primer strand (Keen et al., 2014b; Martínez-Jiménez et al., 2018). An intact ZF is essential for PrimPol's function in vivo (Mourón et al., 2013; Keen et al., 2014a, 2014b; Kobayashi et al., 2016; Schiavone et al., 2016; Šviković et al., 2019). Consequently, the available evidence strongly suggests the primary function of PrimPol in vivo is as a primase not a TLS polymerase (Guilliam and Doherty, 2017).

In avian cells, loss of PrimPol causes damage sensitivity, replication fork slowing and growth arrest after damage (Bailey et al., 2016; Kobayashi et al., 2016). In human cells, deletion of PrimPol does not affect survival after damage but does result in delayed recovery, increased mutagenesis, and sister chromatid exchanges (Bailey et al., 2019). However, loss of PrimPol in human cells lacking Pol $\eta$ or Pol $\zeta$ significantly increases sensitivity to damage (Kobayashi et al., 2016; Bailey et al., 2019). This suggests that PrimPol may function to rescue uncoupled forks when Pol $\delta$ alone or TLS cannot readily bypass a leading-strand obstacle (Figure 5A). In support of this, PrimPol is required for the tolerance of impediments not efficiently bypassed by TLS, including chain-terminating nucleosides (Kobayashi et al., 2016), DNA secondary structures (Schiavone et al., 2016), R-loops (Šviković et al., 2019), cisplatin-induced adducts and hydroxyurea (Quinet et al., 2020), bulky DNA adducts that induce recombination (Piberger et al., 2020), and interstrand crosslinks (ICLs) (González-Acosta et al., 2020, Preprint). Indeed, although Rev1 is involved in bypass of G4quadruplexes (G4s)-DNA secondary structures formed by Hoogsteen base-pairing between guanines-containing long loops (Schiavone et al., 2014), PrimPol is required for tolerance of G4s that are more thermodynamically stable (Schiavone et al., 2016). Moreover, on the fly TLS by Pol $\eta$ was recently found to be the primary mechanism to recouple replisomes in response to UV damage in human fibroblasts, while PrimPol compensated for loss of Pol $\eta$, with the resulting ssDNA gaps filled in by template switching (Benureau et al., 2020, Preprint). Therefore, the relative efficiency of TLS bypass of a given impediment vs. repriming by PrimPol may dictate pathway 


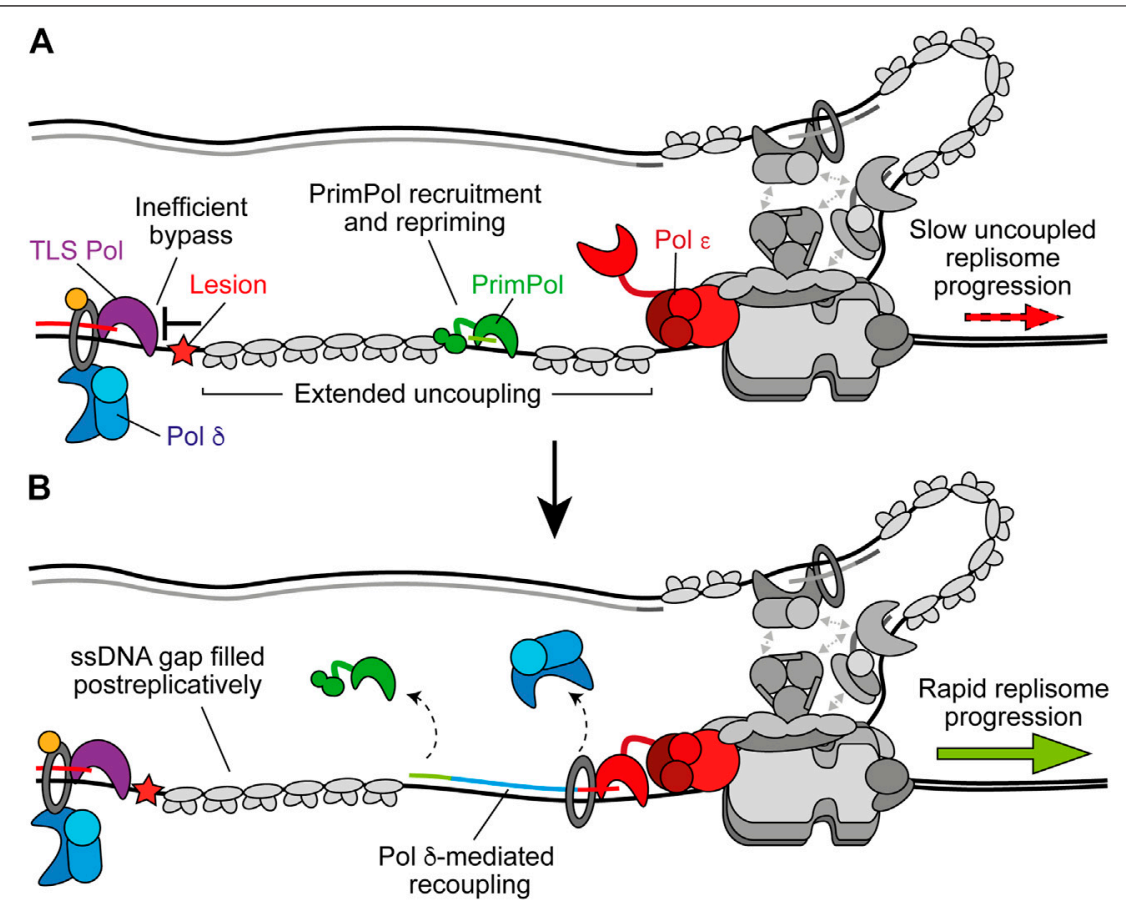

FIGURE 5 | PrimPol-mediated repriming restarts leading-strand synthesis. (A) When replicase switching and on the fly TLS are inefficient at bypassing the leadingstrand impediment, extended uncoupling occurs. This stimulates the recruitment of PrimPol to RPA-coated ssDNA downstream and subsequent repriming. Other replisome components are shown in gray for clarity. (B) Following repriming, Pol $\delta$ may extend the nascent primer to recouple synthesis to Pol $\varepsilon$ and restore rapid replisome progression rates. This leaves behind a ssDNA gap at the site of the lesion which may be filled in postreplicatively by TLS or template switching. Synthesis by Pol $\varepsilon$, Pol $\delta$, and PrimPol are shown in red, blue, and green respectively.

choice. Although importantly, TLS can also function to fill in the ssDNA gap generated after repriming (Figure 5B) (Daigaku et al., 2010; Karras and Jentsch, 2010).

Following repriming, the nascent primer is likely extended by Pol $\delta$ to promote recoupling, as observed following TLS (Figure 5B) (Guilliam and Yeeles, 2020b). Interestingly, PolDIP2 (Pol $\delta$-interacting protein 2, PDIP38) interacts with Pol $\delta$, PCNA, and PrimPol, enhancing the polymerase activity of the latter (Maga et al., 2013; Guilliam et al., 2016; Kasho et al., 2021). PolDIP2 might therefore promote primer extension by PrimPol before also coordinating a switch to $\mathrm{Pol} \delta$ for recoupling. A number of TLS polymerases also interact with PolDIP2 and loss of the protein causes a decrease in TLS in vivo (Tissier et al., 2010; Maga et al., 2013; Tsuda et al., 2019). However, whether PolDIP2 coordinates a switch back to Pol $\delta$ or another aspect of TLS or repriming in higher eukaryotes remains to be determined.

\section{FORK REVERSAL}

Template switching is a homologous recombination (HR)-like mechanism that promotes damage tolerance by using the undamaged nascent strand of the sister chromatid as a template (Branzei and Szakal, 2016). It can function postreplicatively to fill ssDNA gaps on the lagging strand, or following repriming on the leading strand by PrimPol (Piberger et al., 2020). However, template switching may also occur at the replication fork through fork reversal (Berti et al., 2020). Here, the fork regresses by annealing the nascent daughter strands, generating a four-way "chicken foot" structure (Figure 6A). This may allow extension of the nascent leading strand by using the undamaged nascent lagging strand as a template (Figure 6B), or place the damaged parental strand in a dsDNA context to permit canonical repair before fork restart (Figure 6C) (Berti and Vindigni, 2016).

Fork reversal requires Rad51 which binds ssDNA to promote strand invasion during HR (Scully et al., 2019) and partially replaces RPA on the ssDNA exposed by uncoupling, or nascent strand resection, to promote reversal (Berti et al., 2020). How these short stretches of Rad51 separated by RPA-termed metastable Rad51 filaments-are able to promote fork reversal is not currently clear (Berti et al., 2020). However, intriguingly this is not dependent on the enzymatic strand exchange activity of Rad51 (Mason et al., 2019). Additionally, following monoubiquitination by Rad6-Rad18, PCNA can be polyubiquitylated (Ripley et al., 2020). In yeast, Rad5 serves as the E3 ubiquitin-ligase (Hoege et al., 2002) and also has helicase activity that can directly reverse forks (Blastyák et al., 2007). Humans have two Rad5-related proteins, HLTF and SHPRH, both of which can polyubiquitylate monoubiquitinated PCNA, with HLTF shown to catalyse fork reversal in vitro (Unk et al., 2006; Blastyák et al., 2010; Kile et al., 2015). Two additional translocases also mediate fork reversal in humans, ZRANB3 and SMARCAL1, which are recruited by polyubiquitylated PCNA 


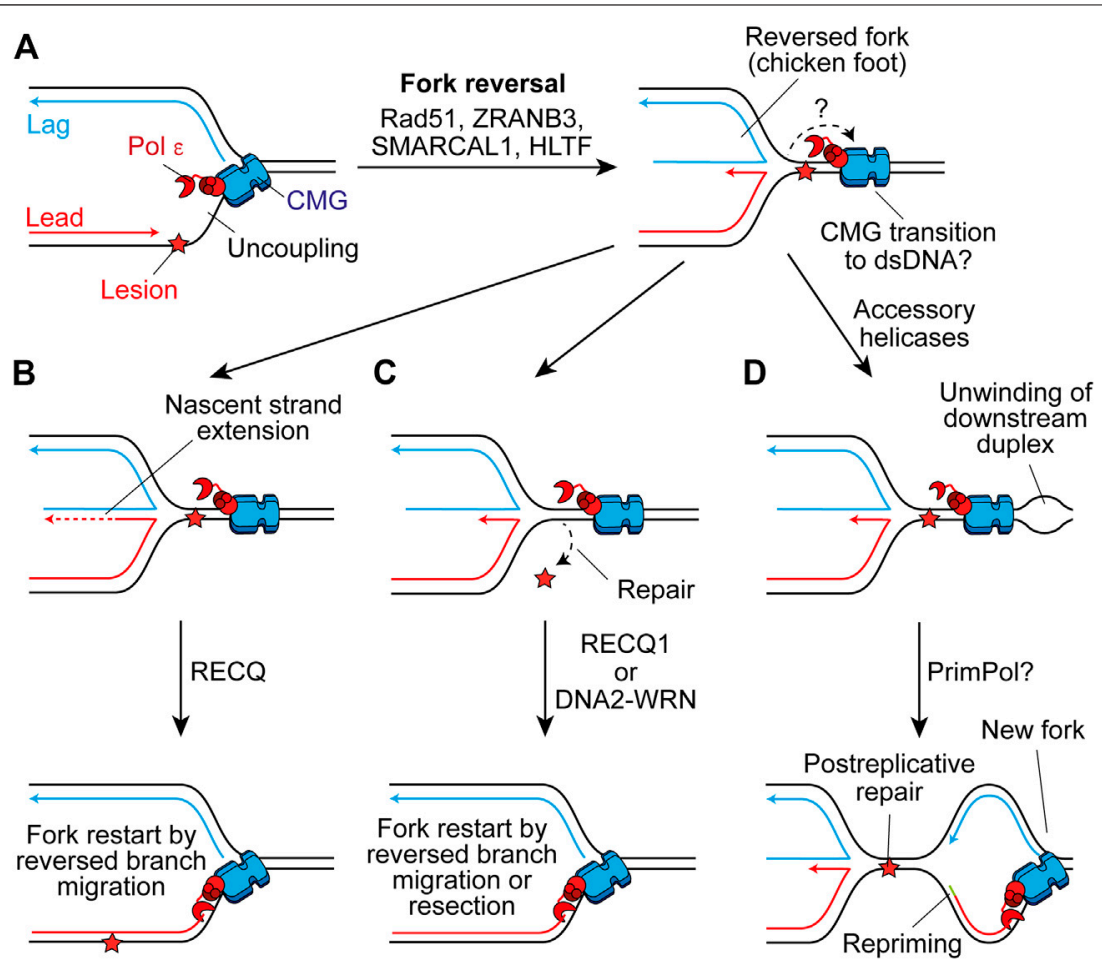

FIGURE 6 | Fork reversal may maintain or restart replisome progression past leading-strand impediments. (A) Following uncoupling, fork reversal can be catalysed by Rad51, ZRANB3, SMARCAL1, and HLTF to generate a four-way chicken foot structure in a process which is not currently well understood. The fate of CMG here is not clear, one possibility is that it transitions on to duplex DNA downstream of the lesion. (B) The nascent lagging strand could serve as a template for extension of the nascent leading strand. Subsequent reversed branch migration, catalysed by RECQ1, may support fork restart if the extended $3^{\prime}$ end of the nascent leading strand is relocated downstream of the lesion. CMG may then transition back onto ssDNA to restart canonical replication. (C) Alternatively, fork reversal may place the lesion in a dsDNA context to permit repair, before resetting of the fork by RECQ1 or resection of nascent strands by DNA2-WRN. Again, CMG may transition back onto ssDNA to restart rapid fork progression. (D) Another possibility is that accessory helicases unwind the parental duplex downstream of the impediment following fork reversal. This may allow the establishment of a new replication fork which would require repriming of the leading strand. This would leave the chicken foot structure behind the new fork to be resolved following postreplicative repair of the lesion.

and RPA-ssDNA respectively (Ciccia et al., 2012; Bétous et al., 2013; Kolinjivadi et al., 2017; Taglialatela et al., 2017). These translocases fulfill non-redundant roles and might therefore contribute to different steps or be required in different contexts (Berti et al., 2020).

Although the generation of reversed forks does not require stable Rad51 filaments, BRCA2-mediated stable Rad51 filaments are required to protect reversed forks from nucleolytic degradation following their formation (Schlacher et al., 2011; Berti et al., 2020). Forks can subsequently be restarted by unwinding and controlled resection by WRNDNA2 (Thangavel et al., 2015) or through reversed-branch migration by RECQ1 (Figures 6B,C) (Berti et al., 2013). Alternatively, a protected reversed fork could await resolution by the arrival of the convergent fork. However, prolonged fork reversal or de-protection can lead to processing by structure specific endonucleases, producing a broken fork (Berti et al., 2020). This can be rescued by break-induced replication, whereby strand invasion by the broken parental strand restarts replication but in an error prone and conservative manner that does not rely on a canonical replisome (Kramara et al., 2018).
Reversed forks have been directly detected using transmission electron microscopy (TEM) in human cells treated with a range of genotoxic agents, suggesting it is a universal response to replication stress (Zellweger et al., 2015). In support, fork reversal has been observed as an ATR-dependent global response to ICLs, even at forks not directly challenged by damage, to slow replication and promote repair (Mutreja et al., 2018). However, ATR phosphorylation of SMARCAL1 has also been shown to limit fork remodeling (Couch et al., 2013). Moreover, a recent study of human fibroblasts failed to detect any evidence of fork reversal by TEM in response to UV damage, even in the absence of $\mathrm{Pol} \eta$, with repriming instead compensating for loss of TLS (Benureau et al., 2020, Preprint). In yeast, reversed forks were observed in repriming (Fumasoni et al., 2015) and checkpoint (Sogo et al., 2002; Lopes et al., 2006) mutants in response to bulky DNA lesions. A recent report showed that ATR-dependent up-regulation of PrimPol in cancer cells following multiple doses of cisplatin treatment suppresses fork reversal, revealing a competition between the two mechanisms (Quinet et al., 2020). This may be because PrimPol limits uncoupling and RPA-ssDNA is a prerequisite for fork reversal (Zellweger et al., 2015). Therefore, fork reversal and repriming 


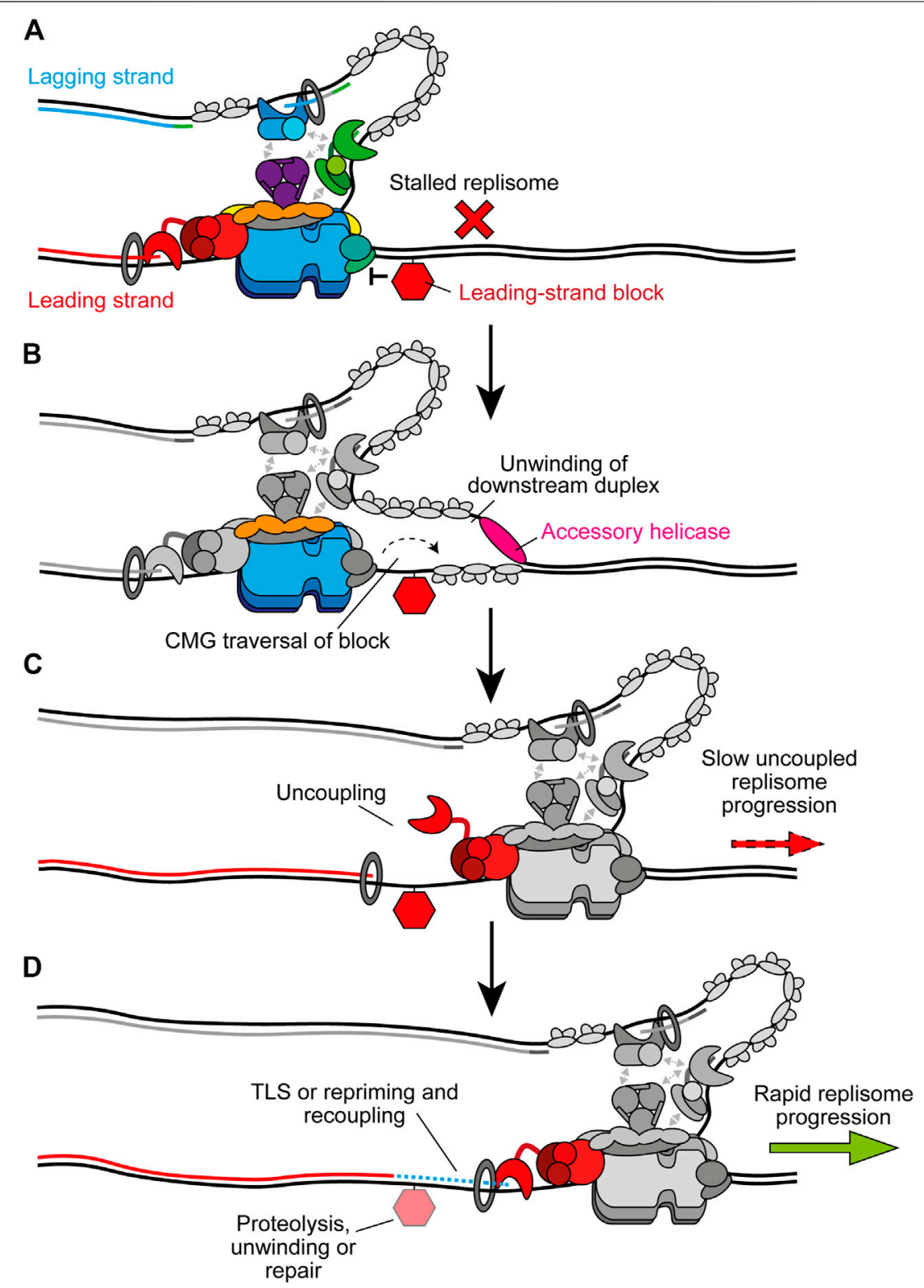

FIGURE 7 | Bypass of CMG-blocking impediments on the leading strand. (A) Bulky leading-strand impediments including DPCs, ICLs, and preformed G4s can pose a block to CMG translocation, causing stalling of the replisome. (B) CMG traversal of the impediment is aided by transient opening of the MCM2-7 ring and accessory helicases which unwind the downstream parental duplex. The specific accessory helicase involved depends on the nature of the lesion, see text for details. Note that the accessory helicase may act on either the leading or lagging strand. Other replisome components are greyed out for clarity. (C) Following CMG traversal of the impediment, the replisome is uncoupled and fork progression is slow. (D) Recoupling first requires resolution of the impediment and/or extension of the leading strand past the damage site. Alternatively, leading-strand synthesis may be reinitiated downstream by repriming before postreplicative repair of the damage. In the case of DPCs, proteolysis occurs before TLS and recoupling. G4s are unwound by FANCJ to promote bypass and recoupling. ICL bypass requires PrimPolmediated repriming before recoupling, leaving an $\mathrm{X}$-shaped structure for postreplicative repair. Upon recoupling, rapid fork rates resume. Recoupling is indicated by a blue dotted line and Pol $\varepsilon$ synthesis is shown in red.

likely compete when on the fly TLS cannot promote efficient bypass, with cell type, the nature of the replication impediment, and complex ATR-orchestrated responses potentially dictating which pathway is favored.

The precise details of fork reversal remain to be determined. In particular, it is not clear what happens to the replisome during this process. In bacteriophage $\mathrm{T} 4$, the replicative helicase must dissociate to permit remodeling of the fork (Manosas et al., 2012). However, in eukaryotes reloading of MCMs is inhibited in S phase to prevent re-replication (Chen and Bell, 2011; Frigola et al., 2013). To function as a mechanism to restart or maintain progression of a replisome, fork reversal must not cause dissociation of CMG. One possibility is that uncoupled CMG traverses onto dsDNA downstream of the stalled fork junction, 
before moving back onto ssDNA to restart replication when the canonical fork is restored (Figures 6A-C). Single-molecule analyses recently demonstrated that $\mathrm{MCM}$ can transiently open to transition from ssDNA to dsDNA, before diffusing back again to reform a functional replisome (Wasserman et al., 2019). This is not dependent on the Mcm2-5 gate used for loading during origin licensing, but does require $\mathrm{Mcm} 10$ which is essential for CMG activation. Here, Mcm10 may prevent dissociation of CMG upon opening of the ssDNA gate. However, $\mathrm{Mcm} 10$ also inhibits fork reversal by SMARCAL1 in vitro (Mayle et al., 2019). Moreover, in Xenopus egg extracts when CMG runs onto dsDNA at a lagging-strand nick it is ubiquitylated and removed by the same pathway used during termination (Vrtis et al., 2021). It is currently unclear how CMG encircling dsDNA to permit fork reversal would resist unloading by the same mechanism. Alternatively, CMG may rapidly reengage ssDNA downstream of the stalled fork junction, aided by accessory helicases (Figure 6D). Such a mechanism would require repriming and leave behind the chicken foot structure for resolution in a postreplicative manner (Berti et al., 2020). In either case, it is unclear what would trigger uncoupled CMG to transition from ssDNA to dsDNA. Indeed, in reconstitution experiments uncoupled CMG continues to unwind the parental template for multiple $\mathrm{kb}$ downstream of a CPD (Taylor and Yeeles, 2018, 2019).

\section{TRAVERSAL OF IMPEDIMENTS TO CMG TRANSLOCATION}

Instead of causing uncoupling, bulkier replication impediments can directly block CMG translocation (Figure 7A). One example are DNA-protein crosslinks (DPCs), chromatin-bound proteins which are covalently crosslinked to DNA by chemotherapeutics or endogenous aldehydes (Stingele et al., 2017). Since dsDNA enters CMG a short distance, with strand separation occurring at the bottom of the ZF sub-ring (Yuan et al., 2020a; Baretić et al., 2020), DPCs might be expected to stall replisomes regardless of which template strand they are located in. However, in Xenopus extracts replisome progression is stalled much more significantly by a streptavidin block in the leading strand compared to the lagging strand (Fu et al., 2011). Purified yeast CMG is stalled by a streptavidin block located in either strand (Langston and O'Donnell, 2017), but can bypass the lagging-strand block in the presence of Mcm10 (Langston et al., 2017). Here, Mcm10 is hypothesised to bind the $\mathrm{N}$ tier of CMG to isomerise it to a steric exclusion model capable of bypassing impediments on the lagging strand (Langston et al., 2017). It is unknown if $\mathrm{Mcm} 10$ remains stably bound to the replisome following initiation or if it interacts dynamically when required.

Recent experiments in Xenopus extracts have revealed that the accessory helicase RTEL1 also stimulates bypass of a lagging strand DPC, although bypass still occurs in its absence (Sparks et al., 2019). Meanwhile, CMG stalling at a leading strand DPC is more extended and more sensitive to loss of RTEL1. Here, RTEL1 generates ssDNA downstream of stalled CMG to facilitate DPC bypass by transient opening of the $\mathrm{Mcm}$ 2-7 ring, potentially aided by Mcm10 (Figure 7B) (Sparks et al., 2019; Wasserman et al., 2019). Single-molecule experiments confirmed that CMG bypass occurs before DPC degradation by the specialised protease SPTRN/ DVC1 (Wss1 in yeast) (Sparks et al., 2019). Interestingly, upon collision the replisome facilitates ubiquitylation of the DPC by the E3 ubiquitin ligase TRAIP to promote proteolysis after CMG bypass. Ubiquitylation of the DPC also aids CMG bypass (Sparks et al., 2019). TRAIP is proposed to bind the leading edge of the replisome to fulfill this role which also positions it to ubiquitylate an adjacent CMG after fork convergence at an ICL (Wu et al., 2019, 2021). Following bypass, CMG progression is initially slow due to uncoupling (Figure 7C). TLS after DPC proteolysis subsequently recouples the fork (Figure 7D) (Duxin et al., 2014; Sparks et al., 2019).

ICLs also represent a block to replisome progression. In Xenopus extracts, ICL repair occurs following fork convergence at the lesion (Zhang et al., 2015). Here, TRAIP-dependent ubiquitylation of CMG stimulates recruitment of the NEIL3 glycosylase to resolve psoralen and abasic ICLs (Wu et al., 2021). If NEIL3 fails to unhook the crosslink, continued ubiquitylation of CMG by TRAIP triggers replisome disassembly to permit repair of the ICL by the Fanconi anemia pathway (Wu et al., 2021). Since both pathways depend on fork convergence, they cannot be considered as mechanisms to maintain progression of individual replisomes. However, studies in avian and mammalian cells suggest CMG can also traverse ICLs and maintain progression via an alternative mechanism (Huang et al., 2013, 2019; Ling et al., 2016; Mutreja et al., 2018). Here, FANCM associates with CMG in a FANCD2 and ATR-signalling dependent manner which triggers release of GINS from the replisome (Huang et al., 2019). FANCM translocase activity and loss of GINS may then promote opening of the Mcm2-7 ring and allow traversal of the ICL. FANCM also interacts with BTR (BLM/TOP3A/RMI1-2), and BLM helicase activity is important for ICL traverse (Ling et al., 2016). BLM might therefore play an analogous role to RTEL1 during DPC bypass by unwinding the parental strands downstream of the ICL (Ling et al., 2016). BTR also interacts with both RPA (Wu et al., 2018) and PrimPol (González-Acosta et al., 2020, preprint). Recent work demonstrated that PrimPol participates in ICL bypass, likely by reinitiating leading-strand synthesis to restore rapid fork rates (González-Acosta et al., 2020, preprint). Traversal would leave the X-shaped ICL structure behind the re-established replication fork to be repaired by the Fanconi anaemia pathway in a postreplicative manner (Lopez-Martinez et al., 2016).

Accessory helicases also assist the replisome in bypassing preformed G4 structures that can stall CMG when present in the leading-strand template (Lerner and Sale, 2019). Experiments in Xenopus extracts have recently delineated a mechanism for G4 bypass similar to that employed for DPC traversal (Sato et al., 2020, preprint). Following CMG collision with a G4, the accessory helicase DHX36 unwinds the parental duplex downstream of the structure. This allows CMG to bypass the G4 without unwinding through opening of the Mcm2-7 ring (Sparks et al., 2019; Wasserman et al., 2019). A second helicase, FANCJ, then assists G4 unwinding to permit recoupling of the leading strand to the replisome. There is partial redundancy between these two helicases for CMG bypass and notably the requirement for both is abolished by a convergent fork (Sato et al., 
2020, preprint). DHX36 and FANCJ likely facilitate bypass in this context instead of RTEL1 due to their high affinity for G4s (Wu et al., 2008; Giri et al., 2011). G4s can also be bypassed by another recently identified mechanism (Lerner et al., 2020). Here, the replisome plays an active role in G4 resolution facilitated by a DNA binding domain in Timeless that has a strong preference for G4s. This allows Timeless to detect G4s in the vicinity of the replisome and promote their unwinding by DDX11, an accessory helicase that interacts with and is stimulated by Timeless (Lerner et al., 2020). The recent structure of Tof1, the yeast homologue of Timeless, in complex with CMG revealed that it binds ahead of the replisome, positioning it in an ideal location to detect G4s in the unwound parental duplex (Baretic et al., 2020). Importantly, G4s can adopt many different conformations and therefore these two mechanisms may favor different subsets of these.

\section{SUMMARY}

Aided by advances in biochemical systems, structural approaches, and single-molecule techniques, our understanding of the architecture of the replisome during unperturbed progression, the response of the replisome to impediments, and the DDT mechanisms employed to overcome these has increased greatly over recent years. In particular, these studies have revealed that DDT mechanisms function not only to relieve polymerase stalling, but also to maintain $\mathrm{CMG}$ translocation in the presence of obstacles previously thought to pose a complete block to template unwinding. Here, the combined action of transient opening of the MCM ring, unwinding of the parental duplex by accessory helicases, and recoupling of the nascent leading strand by classic DDT mechanisms has expanded the range of obstacles considered able to be tolerated by the replisome (Sparks et al., 2019; Wasserman et al., 2019; González-Acosta et al., 2020; Sato et al., 2020, preprint). Meanwhile, the chronology of pathway choice for recoupling leading strand replication is beginning to be deciphered. Recruitment of Pol $\delta$ to the leading strand appears to be the primary mechanism of recoupling (Guilliam and Yeeles, 2020b, 2021). When Pol $\delta$ is unable to fulfill this role, on the fly TLS is triggered by PCNA monoubiquitination and/or Rev1 recruitment

\section{REFERENCES}

Alcasabas, A. A., Osborn, A. J., Bachant, J., Hu, F., Werler, P. J. H., Bousset, K., et al. (2001). Mrc1 Transduces Signals of DNA Replication Stress to Activate Rad53. Nat. Cel Biol. 3, 958-965. doi:10.1038/ncb1101-958

Andersen, P. L., Xu, F., Ziola, B., McGregor, W. G., and Xiao, W. (2011). Sequential Assembly of Translesion DNA Polymerases at UV-Induced DNA Damage Sites. MBoC 22, 2373-2383. doi:10.1091/mbc.E10-120938

Aria, V., and Yeeles, J. T. P. (2019). Mechanism of Bidirectional Leading-Strand Synthesis Establishment at Eukaryotic DNA Replication Origins. Mol. Cel 73, 199-211. doi:10.1016/j.molcel.2018.10.019

Bailey, L. J., Bianchi, J., Hégarat, N., Hochegger, H., and Doherty, A. J. (2016). PrimPol-deficient Cells Exhibit a Pronounced G2 Checkpoint Response Following UV Damage. Cell Cycle 15, 908-918. doi:10.1080/ 15384101.2015.1128597 to prevent prolonged uncoupling (Guilliam and Yeeles, 2020b). At bulkier impediments, or when TLS is inactivated, repriming and fork reversal compete to restore canonical replisome progression (Benureau et al., 2020, preprint; Quinet et al., 2020). In the case of repriming, the resulting ssDNA gaps can be filled in by postreplicative TLS or template switching, as will always occur on the lagging strand.

Many of these mechanisms offer potential targets for future chemotherapeutics. In particular, the role of TLS in both chemoresistance and mutagenesis has made TLS inhibitors a potentially promising future anti-cancer tool (Nayak et al., 2021). Further advances in biochemistry and structural biology will be key to fully understanding the mechanisms for maintaining replisome progression and how these can be exploited for disease treatment.

\section{AUTHOR CONTRIBUTIONS}

TG researched and wrote the manuscript and made the figures.

\section{FUNDING}

This work was supported by a Sir Henry Wellcome Postdoctoral Fellowship from the Wellcome Trust (213596/Z/18/Z). The Yeeles laboratory, of which TG is a member, is supported by the Medical Research Council as part of the United Kingdom Research and Innovation (MRC Grant No. MC_UP_1201/12). This research was funded in whole, or in part, by the Wellcome Trust $(213596 / Z / 18 / Z)$. For the purpose of Open Access, the MRC Laboratory of Molecular Biology has applied a CC BY public copyright licence to any Author Accepted Manuscript (AAM) version arising from this submission.

\section{ACKNOWLEDGMENTS}

I would like to thank M. Jenkyn-Bedford, M. Hodskinson, and R. Westhorpe for critical reading of the manuscript.

Bailey, L. J., Bianchi, J., and Doherty, A. J. (2019). PrimPol Is Required for the Maintenance of Efficient Nuclear and Mitochondrial DNA Replication in Human Cells. Nucleic Acids Res. 47, 4026-4038. doi:10.1093/nar/gkz056

Baranovskiy, A. G., Lada, A. G., Siebler, H. M., Zhang, Y., Pavlov, Y. I., and Tahirov, T. H. (2012). DNA Polymerase $\delta$ and $\zeta$ Switch by Sharing Accessory Subunits of DNA Polymerase $\delta$. J. Biol. Chem. 287, 17281-17287. doi:10.1074/ jbc.M112.351122

Baretić, D., Jenkyn-Bedford, M., Aria, V., Cannone, G., Skehel, M., and Yeeles, J. T. P. (2020). Cryo-EM Structure of the Fork Protection Complex Bound to CMG at a Replication Fork. Mol. Cel 78, 926-e13. doi:10.1016/ j.molcel.2020.04.012

Bétous, R., Couch, F. B., Mason, A. C., Eichman, B. F., Manosas, M., and Cortez, D. (2013). Substrate-Selective Repair and Restart of Replication Forks by DNA Translocases. Cel Rep. 3, 1958-1969. doi:10.1016/j.celrep.2013.05.002

Bell, S. D., and Botchan, M. R. (2013). The Minichromosome Maintenance Replicative Helicase. Cold Spring Harbor Perspect. Biol. 5, a012807. doi: $10.1101 /$ cshperspect.a012807 
Bell, S. P., and Labib, K. (2016). Chromosome Duplication in Saccharomyces cerevisiae. Genetics 203, 1027-1067. doi:10.1534/genetics.115.186452

Benureau, Y., Pouvelle, C., Tavares, E. M., Dupaigne, P., Despras, E., Cam, E. L., et al. (2020). Replication Intermediate Architecture Reveals the Chronology of DNA Damage Tolerance Pathways at UV-Stalled Replication forks in Human Cells. BioRxiv. 11, 23. doi:10.12.33610710.1101/2020.10.12.336107

Bermudez, V. P., Farina, A., Tappin, I., and Hurwitz, J. (2010). Influence of the Human Cohesion Establishment Factor Ctf4/AND-1 on DNA Replication. J. Biol. Chem. 285, 9493-9505. doi:10.1074/jbc.M109.093609

Berti, M., and Vindigni, A. (2016). Replication Stress: Getting Back on Track. Nat. Struct. Mol. Biol. 23, 103-109. doi:10.1038/nsmb.3163

Berti, M., Ray Chaudhuri, A., Thangavel, S., Gomathinayagam, S., Kenig, S., Vujanovic, M., et al. (2013). Human RECQ1 Promotes Restart of Replication forks Reversed by DNA Topoisomerase I Inhibition. Nat. Struct. Mol. Biol. 20, 347-354. doi:10.1038/nsmb.2501

Berti, M., Cortez, D., and Lopes, M. (2020). The Plasticity of DNA Replication forks in Response to Clinically Relevant Genotoxic Stress. Nat. Rev. Mol. Cel Biol. 21, 633-651. doi:10.1038/s41580-020-0257-5

Bianchi, J., Rudd, S. G., Jozwiakowski, S. K., Bailey, L. J., Soura, V., Taylor, E., et al. (2013). PrimPol Bypasses UV Photoproducts during Eukaryotic Chromosomal DNA Replication. Mol. Cel 52, 566-573. doi:10.1016/j.molcel.2013.10.035

Bienko, M., Green, C. M., Crosetto, N., Rudolf, F., Zapart, G., Coull, B., et al. (2005). Ubiquitin-Binding Domains in Y-Family Polymerases Regulate Translesion Synthesis. Science 310, 1821-1824. doi:10.1126/science.1120615

Blastyák, A., Pintér, L., Unk, I., Prakash, L., Prakash, S., and Haracska, L. (2007). Yeast Rad5 Protein Required for Postreplication Repair Has a DNA Helicase Activity Specific for Replication fork Regression. Mol. Cel 28, 167-175. doi:10.1016/j.molcel.2007.07.030

Blastyák, A., Hajdú, I., Unk, I., and Haracska, L. (2010). Role of Double-Stranded DNA Translocase Activity of Human HLTF in Replication of Damaged DNA. Mol. Cel. Biol. 30, 684-693. doi:10.1128/MCB.00863-09

Boehm, E. M., and Washington, M. T. (2016). R.I.P. To the PIP: PCNA-Binding Motif No Longer Considered Specific. BioEssays 38, 1117-1122. doi:10.1002/ bies. 201600116

Boehm, E. M., Spies, M., and Washington, M. T. (2016). PCNA Tool Belts and Polymerase Bridges Form during Translesion Synthesis. Nucleic Acids Res. 44, 8250-8260. doi:10.1093/nar/gkw563

Boudsocq, F., Kokoska, R. J., Plosky, B. S., Vaisman, A., Ling, H., Kunkel, T. A., et al. (2004). Investigating the Role of the Little finger Domain of Y-Family DNA Polymerases in Low Fidelity Synthesis and Translesion Replication. J. Biol. Chem. 279, 32932-32940. doi:10.1074/jbc.M405249200

Branzei, D., and Szakal, B. (2016). DNA Damage Tolerance by Recombination: Molecular Pathways and DNA Structures. DNA Repair 44, 68-75. doi:10.1016/ j.dnarep.2016.05.008

Bulock, C. R., Xing, X., and Shcherbakova, P. V. (2020). DNA Polymerase $\delta$ Proofreads Errors Made by DNA Polymerase $\varepsilon$. Proc. Natl. Acad. Sci. USA 117, 6035-6041. doi:10.1073/pnas.1917624117

Burgers, P. M. J., and Kunkel, T. A. (2017). Eukaryotic DNA Replication Fork. Annu. Rev. Biochem. 86, 417-438. doi:10.1146/annurev-biochem-061516044709

Byun, T. S., Pacek, M., Yee, M., Walter, J. C., and Cimprich, K. A. (2005). Functional Uncoupling of MCM Helicase and DNA Polymerase Activities Activates the ATR-dependent Checkpoint. Genes Dev. 19, 1040-1052. doi:10.1101/gad.1301205

Casas-Delucchi, C. S., Daza-Martin, M., Williams, S. L., and Coster, G. (2021). The Mechanism of Replication Stalling and Recovery within Repetitive DNA. bioRxiv 14, 446729. doi:10.1101/2021.06.02.446729

Chen, S., and Bell, S. P. (2011). CDK Prevents Mcm2-7 Helicase Loading by Inhibiting Cdt1 Interaction with Orc6. Genes Dev. 25, 363-372. doi:10.1101/ gad.2011511

Ciccia, A., Nimonkar, A. V., Hu, Y., Hajdu, I., Achar, Y. J., Izhar, L., et al. (2012). Polyubiquitinated PCNA Recruits the ZRANB3 Translocase to Maintain Genomic Integrity after Replication Stress. Mol. Cel 47, 396-409. doi:10.1016/j.molcel.2012.05.024

Conti, C., Saccà, B., Herrick, J., Lalou, C., Pommier, Y., and Bensimon, A. (2007). Replication Fork Velocities at Adjacent Replication Origins Are Coordinately Modified during DNA Replication in Human Cells. MBoC 18, 3059-3067. doi:10.1091/mbc.E06-08-0689
Costa, A., Ilves, I., Tamberg, N., Petojevic, T., Nogales, E., Botchan, M. R., et al. (2011). The Structural Basis for MCM2-7 Helicase Activation by GINS and Cdc45. Nat. Struct. Mol. Biol. 18, 471-477. doi:10.1038/nsmb.2004

Couch, F. B., Bansbach, C. E., Driscoll, R., Luzwick, J. W., Glick, G. G., Bétous, R., et al. (2013). ATR Phosphorylates SMARCAL1 to Prevent Replication fork Collapse. Genes Dev. 27, 1610-1623. doi:10.1101/gad.214080.113

Courtot, L., Hoffmann, J.-S., and Bergoglio, V. (2018). The Protective Role of Dormant Origins in Response to Replicative Stress. Ijms 19, 3569. doi:10.3390/ ijms 19113569

Cranford, M. T., Chu, A. M., Baguley, J. K., Bauer, R. J., and Trakselis, M. A. (2017). Characterization of a Coupled DNA Replication and Translesion Synthesis Polymerase Supraholoenzyme from Archaea. Nucleic Acids Res. 45, 8329-8340. doi:10.1093/nar/gkx539

Daigaku, Y., Davies, A. A., and Ulrich, H. D. (2010). Ubiquitin-dependent DNA Damage Bypass Is Separable from Genome Replication. Nature 465, 951-955. doi:10.1038/nature09097

Daraba, A., Gali, V. K., Halmai, M., Haracska, L., and Unk, I. (2014). Def1 Promotes the Degradation of Pol3 for Polymerase Exchange to Occur during DNA-Damage-Induced Mutagenesis in Saccharomyces cerevisiae. PLOS Biol. 12, e1001771. doi:10.1371/journal.pbio.1001771

Davies, A. A., Huttner, D., Daigaku, Y., Chen, S., and Ulrich, H. D. (2008). Activation of Ubiquitin-dependent DNA Damage Bypass Is Mediated by Replication Protein a. Mol. Cel 29, 625-636. doi:10.1016/j.molcel.2007.12.016

Devbhandari, S., and Remus, D. (2020). Rad53 Limits CMG Helicase Uncoupling from DNA Synthesis at Replication forks. Nat. Struct. Mol. Biol. 27, 461-471. doi:10.1038/s41594-020-0407-7

Dewar, J. M., and Walter, J. C. (2017). Mechanisms of DNA Replication Termination. Nat. Rev. Mol. Cel Biol. 18, 507-516. doi:10.1038/nrm.2017.42

Douglas, M. E., Ali, F. A., Costa, A., and Diffley, J. F. X. (2018). The Mechanism of Eukaryotic CMG Helicase Activation. Nature 555, 265-268. doi:10.1038/ nature 25787

Duxin, J. P., Dewar, J. M., Yardimci, H., and Walter, J. C. (2014). Repair of a DNAProtein Crosslink by Replication-Coupled Proteolysis. Cell 159, 346-357. doi:10.1016/j.cell.2014.09.024

Edmunds, C. E., Simpson, L. J., and Sale, J. E. (2008). PCNA Ubiquitination and REV1 Define Temporally Distinct Mechanisms for Controlling Translesion Synthesis in the Avian Cell Line DT40. Mol. Cel 30, 519-529. doi:10.1016/ j.molcel.2008.03.024

Eickhoff, P., Kose, H. B., Martino, F., Petojevic, T., Abid Ali, F., Locke, J., et al. (2019). Molecular Basis for ATP-Hydrolysis-Driven DNA Translocation by the CMG Helicase of the Eukaryotic Replisome. Cel Rep. 28, 2673-2688. doi:10.1016/j.celrep.2019.07.104

Enemark, E. J., and Joshua-Tor, L. (2006). Mechanism of DNA Translocation in a Replicative Hexameric Helicase. Nature 442, 270-275. doi:10.1038/ nature 04943

Enemark, E. J., and Joshua-Tor, L. (2008). On Helicases and Other Motor Proteins. Curr. Opin. Struct. Biol. 18, 243-257. doi:10.1016/j.sbi.2008.01.007

Evrin, C., Maman, J. D., Diamante, A., Pellegrini, L., and Labib, K. (2018). Histone H2A-H2B Binding by Pol $\alpha$ in the Eukaryotic Replisome Contributes to the Maintenance of Repressive Chromatin. EMBO J. 37, e99021. doi:10.15252/ embj.201899021

Fleck, O., and Schär, P. (2004). Translesion DNA Synthesis: Little Fingers Teach Tolerance. Curr. Biol. 14, R389-R391. doi:10.1016/j.cub.2004.05.014

Flood, C. L., Rodriguez, G. P., Bao, G., Shockley, A. H., Kow, Y. W., and Crouse, G. F. (2015). Replicative DNA Polymerase $\delta$ but Not $\varepsilon$ Proofreads Errors in Cis and in Trans. PLOS Genet. 11, e1005049. doi:10.1371/journal.pgen.1005049

Freudenthal, B. D., Gakhar, L., Ramaswamy, S., and Washington, M. T. (2010). Structure of Monoubiquitinated PCNA and Implications for Translesion Synthesis and DNA Polymerase Exchange. Nat. Struct. Mol. Biol. 17, 479-484. doi:10.1038/nsmb.1776

Freudenthal, B. D., Beard, W. A., Shock, D. D., and Wilson, S. H. (2013). Observing a DNA Polymerase Choose Right from Wrong. Cell 154, 157-168. doi:10.1016/ j.cell.2013.05.048

Frigola, J., Remus, D., Mehanna, A., and Diffley, J. F. X. (2013). ATPase-dependent Quality Control of DNA Replication Origin Licensing. Nature 495, 339-343. doi:10.1038/nature11920

Fu, Y. V., Yardimci, H., Long, D. T., Guainazzi, A., Bermudez, V. P., Hurwitz, J., et al. (2011). Selective Bypass of a Lagging Strand Roadblock by the 
Eukaryotic Replicative DNA Helicase. Cell 146, 931-941. doi:10.1016/ j.cell.2011.07.045

Fumasoni, M., Zwicky, K., Vanoli, F., Lopes, M., and Branzei, D. (2015). Error-Free DNA Damage Tolerance and Sister Chromatid Proximity during DNA Replication Rely on the Pola/Primase/Ctf4 Complex. Mol. Cel 57, 812-823. doi:10.1016/j.molcel.2014.12.038

Gambus, A., Jones, R. C., Sanchez-Diaz, A., Kanemaki, M., van Deursen, F., Edmondson, R. D., et al. (2006). GINS Maintains Association of Cdc45 with MCM in Replisome Progression Complexes at Eukaryotic DNA Replication forks. Nat. Cel Biol. 8, 358-366. doi:10.1038/ncb1382

Gambus, A., van Deursen, F., Polychronopoulos, D., Foltman, M., Jones, R. C., Edmondson, R. D., et al. (2009). A Key Role for Ctf4 in Coupling the MCM2-7 Helicase to DNA Polymerase a within the Eukaryotic Replisome. EMBO J. 28, 2992-3004. doi:10.1038/emboj.2009.226

Gan, H., Yu, C., Devbhandari, S., Sharma, S., Han, J., Chabes, A., et al. (2017). Checkpoint Kinase Rad53 Couples Leading- and Lagging-Strand DNA Synthesis under Replication Stress. Mol. Cel 68, 446-455.e3. doi:10.1016/ j.molcel.2017.09.018

Gan, H., Serra-Cardona, A., Hua, X., Zhou, H., Labib, K., Yu, C., et al. (2018). The Mcm2-Ctf4-Pol $\alpha$ Axis Facilitates Parental Histone H3-H4 Transfer to Lagging Strands. Mol. Cel 72, 140-151.e3. doi:10.1016/j.molcel.2018.09.001

Gao, Y., Cui, Y., Fox, T., Lin, S., Wang, H., de Val, N., et al. (2019). Structures and Operating Principles of the Replisome. Science 363, eaav7003. doi:10.1126/ science.aav7003

Garbacz, M. A., Lujan, S. A., Burkholder, A. B., Cox, P. B., Wu, Q., Zhou, Z.-X., et al. (2018). Evidence that DNA Polymerase $\delta$ Contributes to Initiating Leading Strand DNA Replication in Saccharomyces cerevisiae. Nat. Commun. 9, 858. doi:10.1038/s41467-018-03270-4

García-Gómez, S., Reyes, A., Martínez-Jiménez, M. I., Chocrón, E. S., Mourón, S., Terrados, G., et al. (2013). PrimPol, an Archaic Primase/polymerase Operating in Human Cells. Mol. Cel 52, 541-553. doi:10.1016/j.molcel.2013.09.025

Garg, P., and Burgers, P. M. (2005). Ubiquitinated Proliferating Cell Nuclear Antigen Activates Translesion DNA Polymerases and REV1. Proc. Natl. Acad. Sci. 102, 18361-18366. doi:10.1073/pnas.0505949102

Georgescu, R. E., Langston, L., Yao, N. Y., Yurieva, O., Zhang, D., Finkelstein, J., et al. (2014). Mechanism of Asymmetric Polymerase Assembly at the Eukaryotic Replication fork. Nat. Struct. Mol. Biol. 21, 664-670. doi:10.1038/nsmb.2851

Georgescu, R., Yuan, Z., Bai, L., de Luna Almeida Santos, R., Sun, J., Zhang, D., et al. (2017). Structure of Eukaryotic CMG Helicase at a Replication fork and Implications to Replisome Architecture and Origin Initiation. Proc. Natl. Acad. Sci. USA 114, E697-E706. doi:10.1073/pnas.1620500114

Giri, B., Smaldino, P. J., Thys, R. G., Creacy, S. D., Routh, E. D., Hantgan, R. R., et al. (2011). G4 Resolvase 1 Tightly Binds and Unwinds Unimolecular G4-DNA. Nucleic Acids Res. 39, 7161-7178. doi:10.1093/nar/gkr234

González-Acosta, D., Blanco-Romero, E., Mutreja, K., Llanos, S., Míguez, S., García, F., et al. (2020). PrimPol Primase Mediates Replication Traverse of DNA Interstrand Crosslinks, bioRxiv 17, 104729. doi:10.1101/2020.05.19.104729

Goswami, P., Abid Ali, F., Douglas, M. E., Locke, J., Purkiss, A., Janska, A., et al. (2018). Structure of DNA-CMG-Pol Epsilon Elucidates the Roles of the Noncatalytic Polymerase Modules in the Eukaryotic Replisome. Nat. Commun. 9, 1-13. doi:10.1038/s41467-018-07417-1

Guan, C., Li, J., Sun, D., Liu, Y., and Liang, H. (2017). The Structure and Polymerase-Recognition Mechanism of the Crucial Adaptor Protein AND-1 in the Human Replisome. J. Biol. Chem. 292, 9627-9636. doi:10.1074/ jbc.M116.758524

Guilliam, T., and Doherty, A. (2017). PrimPol-Prime Time to Reprime. Genes 8, 20. doi:10.3390/genes8010020

Guilliam, T. A., and Yeeles, J. T. P. (2020a). An Updated Perspective on the Polymerase Division of Labor during Eukaryotic DNA Replication. Crit. Rev. Biochem. Mol. Biol. 55, 469-481. doi:10.1080/10409238.2020.1811630

Guilliam, T. A., and Yeeles, J. T. P. (2020b). Reconstitution of Translesion Synthesis Reveals a Mechanism of Eukaryotic DNA Replication Restart. Nat. Struct. Mol. Biol. 27, 450-460. doi:10.1038/s41594-020-0418-4

Guilliam, T. A., and Yeeles, J. T. (2021). The Eukaryotic Replisome Tolerates Leading-strand Base Damage by Replicase Switching. EMBO J. 40, e107037. doi:10.15252/embj.2020107037
Guilliam, T. A., Jozwiakowski, S. K., Ehlinger, A., Barnes, R. P., Rudd, S. G., Bailey, L. J., et al. (2015a). Human PrimPol Is a Highly Error-Prone Polymerase Regulated by Single-Stranded DNA Binding Proteins. Nucleic Acids Res. 43, 1056-1068. doi:10.1093/nar/gku1321

Guilliam, T. A., Keen, B. A., Brissett, N. C., and Doherty, A. J. (2015b). Primasepolymerases Are a Functionally Diverse Superfamily of Replication and Repair Enzymes. Nucleic Acids Res. 43, 6651-6664. doi:10.1093/nar/gkv625

Guilliam, T. A., Bailey, L. J., Brissett, N. C., and Doherty, A. J. (2016). PolDIP2 Interacts with Human PrimPol and Enhances its DNA Polymerase Activities. Nucleic Acids Res. 44, 3317-3329. doi:10.1093/nar/gkw175

Guilliam, T. A., Brissett, N. C., Ehlinger, A., Keen, B. A., Kolesar, P., Taylor, E. M., et al. (2017). Molecular Basis for PrimPol Recruitment to Replication forks by RPA. Nat. Commun. 8, 15222. doi:10.1038/ncomms15222

Guo, C., Sonoda, E., Tang, T.-S., Parker, J. L., Bielen, A. B., Takeda, S., et al. (2006). REV1 Protein Interacts with PCNA: Significance of the REV1 BRCT Domain In Vitro and In Vivo. Mol. Cel 23, 265-271. doi:10.1016/ j.molcel.2006.05.038

Haracska, L., Unk, I., Johnson, R. E., Johansson, E., Burgers, P. M. J., Prakash, S., et al. (2001). Roles of Yeast DNA Polymerases delta and Zeta and of Rev1 in the Bypass of Abasic Sites. Genes Dev. 15, 945-954. doi:10.1101/gad.882301

Hirota, K., Yoshikiyo, K., Guilbaud, G., Tsurimoto, T., Murai, J., Tsuda, M., et al. (2015). The POLD3 Subunit of DNA Polymerase $\delta$ Can Promote Translesion Synthesis Independently of DNA Polymerase $\zeta$. Nucleic Acids Res. 43, 1671-1683. doi:10.1093/nar/gkv023

Hirota, K., Tsuda, M., Mohiuddin, T., Tsurimoto, T., Cohen, I. S., Livneh, Z., et al. (2016). In Vivoevidence for Translesion Synthesis by the Replicative DNA Polymerase $\delta$. Nucleic Acids Res. 44, 7242-7250. doi:10.1093/nar/gkw439

Hoege, C., Pfander, B., Moldovan, G.-L., Pyrowolakis, G., and Jentsch, S. (2002). RAD6-dependent DNA Repair Is Linked to Modification of PCNA by Ubiquitin and SUMO. Nature 419, 135-141. doi:10.1038/nature00991

Huang, M. E., Le Douarin, B., Henry, C., and Galibert, F. (1999). The Saccharomyces cerevisiae Protein YJR043C (Pol32) Interacts with the Catalytic Subunit of DNA Polymerase a and Is Required for Cell Cycle Progression in G2/M. Mol. Gen. Genet. 260, 541-550. doi:10.1007/ s004380050927

Huang, J., Liu, S., Bellani, M. A., Thazhathveetil, A. K., Ling, C., de Winter, J. P., et al. (2013). The DNA Translocase FANCM/MHF Promotes Replication Traverse of DNA Interstrand Crosslinks. Mol. Cel 52, 434-446. doi:10.1016/ j.molcel.2013.09.021

Huang, J., Zhang, J., Bellani, M. A., Pokharel, D., Gichimu, J., James, R. C., et al. (2019). Remodeling of Interstrand Crosslink Proximal Replisomes Is Dependent on ATR, FANCM, and FANCD2. Cel Rep. 27, 1794-1808.e5. doi:10.1016/j.celrep.2019.04.032

Ilves, I., Petojevic, T., Pesavento, J. J., and Botchan, M. R. (2010). Activation of the MCM2-7 Helicase by Association with Cdc45 and GINS Proteins. Mol. Cel 37, 247-258. doi:10.1016/j.molcel.2009.12.030

Indiani, C., McInerney, P., Georgescu, R., Goodman, M. F., and O'Donnell, M. (2005). A Sliding-Clamp Toolbelt Binds High- and Low-Fidelity DNA Polymerases Simultaneously. Mol. Cel 19, 805-815. doi:10.1016/ j.molcel.2005.08.011

Itsathitphaisarn, O., Wing, R. A., Eliason, W. K., Wang, J., and Steitz, T. A. (2012). The Hexameric Helicase DnaB Adopts a Nonplanar Conformation during Translocation. Cell 151, 267-277. doi:10.1016/j.cell.2012.09.014

Jain, R., Rice, W. J., Malik, R., Johnson, R. E., Prakash, L., Prakash, S., et al. (2019). Cryo-EM Structure and Dynamics of Eukaryotic DNA Polymerase $\delta$ Holoenzyme. Nat. Struct. Mol. Biol. 26, 955-962. doi:10.1038/s41594-0190305-z

Johansson, E., Garg, P., and Burgers, P. M. J. (2004). The Pol32 Subunit of DNA Polymerase $\delta$ Contains Separable Domains for Processive Replication and Proliferating Cell Nuclear Antigen (PCNA) Binding. J. Biol. Chem. 279, 1907-1915. doi:10.1074/jbc.M310362200

Johnson, R. E., Washington, M. T., Haracska, L., Prakash, S., and Prakash, L. (2000). Eukaryotic Polymerases I and $\zeta$ Act Sequentially to Bypass DNA Lesions. Nature 406, 1015-1019. doi:10.1038/35023030

Johnson, R. E., Prakash, L., and Prakash, S. (2012). Pol31 and Pol32 Subunits of Yeast DNA Polymerase Are Also Essential Subunits of DNA Polymerase. Proc. Natl. Acad. Sci. 109, 12455-12460. doi:10.1073/pnas.1206052109 
Johnson, K. A. (2008). Role of Induced Fit in Enzyme Specificity: A Molecular Forward/Reverse Switch. J. Biol. Chem. 283, 26297-26301. doi:10.1074/ jbc.R800034200

Kainuma-Kuroda, R., and Okazaki, R. (1975). Mechanism of DNA Chain Growth. J. Mol. Biol. 94, 213-228. doi:10.1016/0022-2836(75)90079-0

Kannouche, P. L., Wing, J., and Lehmann, A. R. (2004). Interaction of Human DNA Polymerase $\eta$ with Monoubiquitinated PCNA. Mol. Cel 14, 491-500. doi:10.1016/s1097-2765(04)00259-x

Kapadia, N., El-Hajj, Z. W., Zheng, H., Beattie, T. R., Yu, A., and Reyes-Lamothe, R. (2020). Processive Activity of Replicative DNA Polymerases in the Replisome of Live Eukaryotic Cells. Mol. Cel 80, 114-126.e8. doi:10.1016/ j.molcel.2020.08.014

Karras, G. I., and Jentsch, S. (2010). The RAD6 DNA Damage Tolerance Pathway Operates Uncoupled from the Replication Fork and Is Functional beyond S Phase. Cell 141, 255-267. doi:10.1016/j.cell.2010.02.028

Kasho, K., Stojkovič, G., Velázquez-Ruiz, C., Martínez-Jiménez, M. I., Doimo, M., Laurent, T., et al. (2021). A Unique Arginine Cluster in PolDIP2 Enhances Nucleotide Binding and DNA Synthesis by PrimPol. Nucleic Acids Res. 49, 2179-2191. doi:10.1093/nar/gkab049

Kath, J. E., Chang, S., Scotland, M. K., Wilbertz, J. H., Jergic, S., Dixon, N. E., et al. (2016). Exchange betweenEscherichia Colipolymerases II and III on a Processivity Clamp. Nucleic Acids Res. 44, 1681-1690. doi:10.1093/nar/ gkv1375

Keen, B. A., Bailey, L. J., Jozwiakowski, S. K., and Doherty, A. J. (2014a). Human PrimPol Mutation Associated with High Myopia Has a DNA Replication Defect. Nucleic Acids Res. 42, 12102-12111. doi:10.1093/nar/gku879

Keen, B. A., Jozwiakowski, S. K., Bailey, L. J., Bianchi, J., and Doherty, A. J. (2014b). Molecular Dissection of the Domain Architecture and Catalytic Activities of Human PrimPol. Nucleic Acids Res. 42, 5830-5845. doi:10.1093/ nar/gku214

Kile, A. C., Chavez, D. A., Bacal, J., Eldirany, S., Korzhnev, D. M., Bezsonova, I., et al. (2015). HLTF's Ancient HIRAN Domain Binds 3' DNA Ends to Drive Replication Fork Reversal. Mol. Cel 58, 1090-1100. doi:10.1016/ j.molcel.2015.05.013

Kilkenny, M. L., Simon, A. C., Mainwaring, J., Wirthensohn, D., Holzer, S., and Pellegrini, L. (2017). The Human CTF4-Orthologue AND-1 Interacts with DNA Polymerase a/primase via its Unique C-Terminal HMG Box. Open Biol. 7, 170217. doi:10.1098/rsob.170217

Kobayashi, K., Guilliam, T. A., Tsuda, M., Yamamoto, J., Bailey, L. J., Iwai, S., et al. (2016). Repriming by PrimPol Is Critical for DNA Replication Restart Downstream of Lesions and Chain-Terminating Nucleosides. Cell Cycle 15, 1997-2008. doi:10.1080/15384101.2016.1191711

Kolinjivadi, A. M., Sannino, V., De Antoni, A., Zadorozhny, K., Kilkenny, M., Técher, H., et al. (2017). Smarcal1-Mediated Fork Reversal Triggers Mre11dependent Degradation of Nascent DNA in the Absence of Brca2 and Stable Rad51 Nucleofilaments. Mol. Cel 67, 867-881.e7. doi:10.1016/ j.molcel.2017.07.001

Kramara, J., Osia, B., and Malkova, A. (2018). Break-Induced Replication: The where, the Why, and the How. Trends Genet. 34, 518-531. doi:10.1016/ j.tig.2018.04.002

Kurat, C. F., Yeeles, J. T. P., Patel, H., Early, A., and Diffley, J. F. X. (2017). Chromatin Controls DNA Replication Origin Selection, Lagging-Strand Synthesis, and Replication Fork Rates. Mol. Cel 65, 117-130. doi:10.1016/ j.molcel.2016.11.016

Lancey, C., Tehseen, M., Raducanu, V.-S., Rashid, F., Merino, N., Ragan, T. J., et al. (2020a). Structure of the Processive Human Pol $\delta$ Holoenzyme. Nat. Commun. 11, 1109. doi:10.1038/s41467-020-14898-6

Lancey, C., Tehseen, M., Takahashi, M., Sobhy, M. A., Ragan, T. J., Crehuet, R., et al. (2020b). Cryo-EM Structure of Pol k-DNA-PCNA Holoenzyme and Implications for Polymerase Switching in DNA Lesion Bypass. bioRxiv 14, 171. doi:10.1101/2020.07.10.196956

Langston, L. D., and O'Donnell, M. (2008). DNA Polymerase $\delta$ Is Highly Processive with Proliferating Cell Nuclear Antigen and Undergoes Collision Release upon Completing DNA. J. Biol. Chem. 283, 29522-29531. doi:10.1074/ jbc.M804488200

Langston, L., and O'Donnell, M. (2017). Action of CMG with Strand-specific DNA Blocks Supports an Internal Unwinding Mode for the Eukaryotic Replicative Helicase. eLife 6, e23449. doi:10.7554/eLife.23449
Langston, L. D., Mayle, R., Schauer, G. D., Yurieva, O., Zhang, D., Yao, N. Y., et al. (2017). Mcm10 Promotes Rapid Isomerization of CMG-DNA for Replisome Bypass of Lagging Strand DNA Blocks. eLife 6, e29118. doi:10.7554/eLife.29118

Lawrence, C. W. (2002). Cellular Roles of DNA Polymerase $\zeta$ and Rev1 Protein. DNA Repair 1, 425-435. doi:10.1016/S1568-7864(02)00038-1

Lerner, L. K., and Sale, J. E. (2019). Replication of G Quadruplex DNA. Genes 10, 95. doi:10.3390/genes 10020095

Lerner, L. K., Holzer, S., Kilkenny, M. L., Šviković, S., Murat, P., Schiavone, D., et al. (2020). Timeless Couples G-quadruplex Detection with Processing by DDX 11 Helicase during DNA Replication. EMBO J. 39, e104185. doi:10.15252/ embj.2019104185

Leung, W., Baxley, R., Moldovan, G.-L., and Bielinsky, A.-K. (2019). Mechanisms of DNA Damage Tolerance: Post-Translational Regulation of PCNA. Genes 10, 10. doi:10.3390/genes 10010010

Lewis, J. S., and Costa, A. (2020). Caught in the Act: Structural Dynamics of Replication Origin Activation and fork Progression. Biochem. Soc. Trans. 48, 1057-1066. doi:10.1042/BST20190998

Lewis, J. S., Spenkelink, L. M., Schauer, G. D., Yurieva, O., Mueller, S. H., Natarajan, V., et al. (2020). Tunability of DNA Polymerase Stability during Eukaryotic DNA Replication. Mol. Cel 77, 17-25.e5. doi:10.1016/j.molcel.2019.10.005

Ling, C., Huang, J., Yan, Z., Li, Y., Ohzeki, M., Ishiai, M., et al. (2016). Bloom Syndrome Complex Promotes FANCM Recruitment to Stalled Replication forks and Facilitates Both Repair and Traverse of DNA Interstrand Crosslinks. Cell Discov 2, 16047. doi:10.1038/celldisc.2016.47

Livneh, Z., Z, O., and Shachar, S. (2010). Multiple Two-Polymerase Mechanisms in Mammalian Translesion DNA Synthesis. Cell CycleTex 9, 729-735. doi:10.4161/cc.9.4.10727

Lopes, M., Foiani, M., and Sogo, J. M. (2006). Multiple Mechanisms Control Chromosome Integrity after Replication Fork Uncoupling and Restart at Irreparable UV Lesions. Mol. Cel 21, 15-27. doi:10.1016/j.molcel.2005.11.015

Lopez-Martinez, D., Liang, C.-C., and Cohn, M. A. (2016). Cellular Response to DNA Interstrand Crosslinks: the Fanconi Anemia Pathway. Cell. Mol. Life Sci. 73, 3097-3114. doi:10.1007/s00018-016-2218-x

Lou, H., Komata, M., Katou, Y., Guan, Z., Reis, C. C., Budd, M., et al. (2008). Mrc1 and DNA Polymerase $\varepsilon$ Function Together in Linking DNA Replication and the S Phase Checkpoint. Mol. Cel 32, 106-117. doi:10.1016/j.molcel.2008.08.020

Maga, G., Crespan, E., Markkanen, E., Imhof, R., Furrer, A., Villani, G., et al. (2013). DNA Polymerase -interacting Protein 2 Is a Processivity Factor for DNA Polymerase during 8-Oxo-7,8-Dihydroguanine Bypass. Proc. Natl. Acad. Sci. 110, 18850-18855. doi:10.1073/pnas.1308760110

Makarova, A. V., Stodola, J. L., and Burgers, P. M. (2012). A Four-Subunit DNA Polymerase $\zeta$ Complex Containing Pol $\delta$ Accessory Subunits Is Essential for PCNA-Mediated Mutagenesis. Nucleic Acids Res. 40, 11618-11626. doi:10.1093/nar/gks948

Malik, R., Kopylov, M., Gomez-Llorente, Y., Jain, R., Johnson, R. E., Prakash, L., et al. (2020). Structure and Mechanism of B-Family DNA Polymerase $\zeta$ Specialized for Translesion DNA Synthesis. Nat. Struct. Mol. Biol. 27, 913-924. doi:10.1038/s41594-020-0476-7

Manosas, M., Perumal, S. K., Croquette, V., and Benkovic, S. J. (2012). Direct Observation of Stalled fork Restart via fork Regression in the T4 Replication System. Science 338, 1217-1220. doi:10.1126/science.1225437

Martínez-Jiménez, M. I., Calvo, P. A., García-Gómez, S., Guerra-González, S., and Blanco, L. (2018). The Zn-finger Domain of Human PrimPol Is Required to Stabilize the Initiating Nucleotide during DNA Priming. Nucleic Acids Res. 46, 4138-4151. doi:10.1093/nar/gky230

Mason, J. M., Chan, Y.-L., Weichselbaum, R. W., and Bishop, D. K. (2019). Nonenzymatic Roles of Human RAD51 at Stalled Replication forks. Nat. Commun. 10, 4410. doi:10.1038/s41467-019-12297-0

Mayle, R., Langston, L., Molloy, K. R., Zhang, D., Chait, B. T., and O’Donnell, M. E. (2019). Mcm10 Has Potent Strand-Annealing Activity and Limits TranslocaseMediated fork Regression. Proc. Natl. Acad. Sci. USA 116, 798-803. doi:10.1073/pnas.1819107116

McClure, A. W., and Diffley, J. F. X. (2021). Rad53 Checkpoint Kinase Regulation of DNA Replication fork Rate via Mrc1 Phosphorylation. bioRxiv 7, 121. doi:10.1101/2021.04.09.439171

McCulloch, S. D., and Kunkel, T. A. (2008). The Fidelity of DNA Synthesis by Eukaryotic Replicative and Translesion Synthesis Polymerases. Cell Res 18, 148-161. doi:10.1038/cr.2008.4 
Meagher, M., Epling, L. B., and Enemark, E. J. (2019). DNA Translocation Mechanism of the MCM Complex and Implications for Replication Initiation. Nat. Commun. 10, 3117. doi:10.1038/s41467-019-11074-3

Mondol, T., Stodola, J. L., Galletto, R., and Burgers, P. M. (2019). PCNA Accelerates the Nucleotide Incorporation Rate by DNA Polymerase $\delta$. Nucleic Acids Res. 47, 1977-1986. doi:10.1093/nar/gky1321

Mourón, S., Rodriguez-Acebes, S., Martínez-Jiménez, M. I., García-Gómez, S., Chocrón, S., Blanco, L., et al. (2013). Repriming of DNA Synthesis at Stalled Replication forks by Human PrimPol. Nat. Struct. Mol. Biol. 20, 1383-1389. doi:10.1038/nsmb.2719

Murakumo, Y., Ogura, Y., Ishii, H., Numata, S.-i., Ichihara, M., Croce, C. M., et al. (2001). Interactions in the Error-Prone Postreplication Repair Proteins hREV1, hREV3, and hREV7. J. Biol. Chem. 276, 35644-35651. doi:10.1074/ jbc.M102051200

Mutreja, K., Krietsch, J., Hess, J., Ursich, S., Berti, M., Roessler, F. K., et al. (2018). ATR-Mediated Global Fork Slowing and Reversal Assist Fork Traverse and Prevent Chromosomal Breakage at DNA Interstrand Cross-Links. Cel Rep. 24, 2629-2642.e5. doi:10.1016/j.celrep.2018.08.019

Nayak, S., Calvo, J. A., and Cantor, S. B. (2021). Targeting Translesion Synthesis (TLS) to Expose Replication Gaps, a Unique Cancer Vulnerability. Expert Opin. Ther. Targets 25, 27-36. doi:10.1080/14728222.2021.1864321

Nelson, J. R., Gibbs, P. E. M., Nowicka, A. M., Hinkle, D. C., and Lawrence, C. W. (2000). Evidence for a Second Function for Saccharomyces cerevisiae Revlp. Mol. Microbiol. 37, 549-554. doi:10.1046/j.1365-2958.2000.01997.x

Ohashi, E., Hanafusa, T., Kamei, K., Song, I., Tomida, J., Hashimoto, H., et al. (2009). Identification of a Novel REV1-Interacting Motif Necessary for DNA Polymerase $\kappa$ Function. Genes Cell Devoted Mol. Cel. Mech. 14, 101-111. doi:10.1111/j.1365-2443.2008.01255.x

On, K. F., Beuron, F., Frith, D., Snijders, A. P., Morris, E. P., and Diffley, J. F. X. (2014). Prereplicative Complexes Assembled In Vitro Support Origindependent and Independent DNA Replication. EMBO J. 33, 605-620. doi:10.1002/embj.201387369

Pardo, B., Crabbé, L., and Pasero, P. (2017). Signaling Pathways of Replication Stress in Yeast. FEMS Yeast Res. 17, fow101. doi:10.1093/femsyr/fow101

Pellegrini, L., and Costa, A. (2016). New Insights into the Mechanism of DNA Duplication by the Eukaryotic Replisome. Trends Biochem. Sci. 41, 859-871. doi:10.1016/j.tibs.2016.07.011

Piberger, A. L., Bowry, A., Kelly, R. D. W., Walker, A. K., González-Acosta, D., Bailey, L. J., et al. (2020). PrimPol-dependent Single-Stranded gap Formation Mediates Homologous Recombination at Bulky DNA Adducts. Nat. Commun. 11, 5863. doi:10.1038/s41467-020-19570-7

Plosky, B. S., Vidal, A. E., de Henestrosa, A. R. F., McLenigan, M. P., McDonald, J. P., Mead, S., et al. (2006). Controlling the Subcellular Localization of DNA Polymerases $\mathrm{I}$ and $\eta$ via Interactions with Ubiquitin. EMBO J. 25, 2847-2855. doi:10.1038/sj.emboj.7601178

Pustovalova, Y., Magalhães, M. T. Q., D’Souza, S., Rizzo, A. A., Korza, G., Walker, G. C., et al. (2016). Interaction between the Rev1 C-Terminal Domain and the PolD3 Subunit of Polל Suggests a Mechanism of Polymerase Exchange upon Rev1/Polל-dependent Translesion Synthesis. Biochemistry 55, 2043-2053. doi:10.1021/acs.biochem.5b01282

Quinet, A., Tirman, S., Jackson, J., Šviković, S., Lemaçon, D., Carvajal-Maldonado, D., et al. (2020). PRIMPOL-Mediated Adaptive Response Suppresses Replication Fork Reversal in BRCA-Deficient Cells. Mol. Cel 77, 461-474.e9. doi:10.1016/j.molcel.2019.10.008

Rechkoblit, O., Gupta, Y. K., Malik, R., Rajashankar, K. R., Johnson, R. E., Prakash, L., et al. (2016). Structure and Mechanism of Human PrimPol, a DNA Polymerase with Primase Activity. Sci. Adv. 2, e1601317. doi:10.1126/ sciadv. 1601317

Reha-Krantz, L. J. (2010). DNA Polymerase Proofreading: Multiple Roles Maintain Genome Stability. Biochim. Biophys. Acta (Bba) - Proteins Proteomics 1804, 1049-1063. doi:10.1016/j.bbapap.2009.06.012

Ripley, B. M., Gildenberg, M. S., and Washington, M. T. (2020). Control of DNA Damage Bypass by Ubiquitylation of PCNA. Genes 11, 138. doi:10.3390/ genes11020138

Robinson, N. P., and Bell, S. D. (2005). Origins of DNA Replication in the Three Domains of Life. FEBS J. 272, 3757-3766. doi:10.1111/j.17424658.2005.04768.x
Ross, A.-L., Simpson, L. J., and Sale, J. E. (2005). Vertebrate DNA Damage Tolerance Requires the C-Terminus but Not BRCT or Transferase Domains of REV1. Nucleic Acids Res. 33, 1280-1289. doi:10.1093/nar/gki279

Rzechorzek, N. J., Hardwick, S. W., Jatikusumo, V. A., Chirgadze, D. Y., and Pellegrini, L. (2020). CryoEM Structures of Human CMG-Atpys-DNA and CMG-AND-1 Complexes. Nucleic Acids Res. 48, 6980-6995. doi:10.1093/nar/ gkaa429

Sale, J. E., Lehmann, A. R., and Woodgate, R. (2012). Y-family DNA Polymerases and Their Role in Tolerance of Cellular DNA Damage. Nat. Rev. Mol. Cel Biol. 13, 141-152. doi:10.1038/nrm3289

Sato, K., Martin-Pintado, N., Post, H., Altelaar, M., and Knipscheer, P. (2020). Multistep Mechanism of DNA Replication-Coupled G-Quadruplex Resolution. bioRxiv 11, 378067. doi:10.1101/2020.11.11.378067

Schauer, G. D., and O’Donnell, M. E. (2017). Quality Control Mechanisms Exclude Incorrect Polymerases from the Eukaryotic Replication fork. Proc. Natl. Acad. Sci. USA 114, 675-680. doi:10.1073/pnas.1619748114

Schiavone, D., Guilbaud, G., Murat, P., Papadopoulou, C., Sarkies, P., Prioleau, M. N., et al. (2014). Determinants of G Quadruplex-induced Epigenetic Instability in REV 1-deficient Cells. EMBO J. 33, 2507-2520. doi:10.15252/ embj.201488398

Schiavone, D., Jozwiakowski, S. K., Romanello, M., Guilbaud, G., Guilliam, T. A., Bailey, L. J., et al. (2016). PrimPol Is Required for Replicative Tolerance of G Quadruplexes in Vertebrate Cells. Mol. Cel 61, 161-169. doi:10.1016/ j.molcel.2015.10.038

Schlacher, K., Christ, N., Siaud, N., Egashira, A., Wu, H., and Jasin, M. (2011). Double-strand Break Repair-independent Role for BRCA2 in Blocking Stalled Replication fork Degradation by MRE11. Cell 145, 529-542. doi:10.1016/ j.cell.2011.03.041

Scully, R., Panday, A., Elango, R., and Willis, N. A. (2019). DNA Double-Strand Break Repair-Pathway Choice in Somatic Mammalian Cells. Nat. Rev. Mol. Cel Biol. 20, 698-714. doi:10.1038/s41580-019-0152-0

Sekedat, M. D., Fenyö, D., Rogers, R. S., Tackett, A. J., Aitchison, J. D., and Chait, B. T. (2010). GINS Motion Reveals Replication fork Progression Is Remarkably Uniform throughout the Yeast Genome. Mol. Syst. Biol. 6, 353. doi:10.1038/ msb. 2010.8

Sengupta, S., van Deursen, F., de Piccoli, G., and Labib, K. (2013). Dpb2 Integrates the Leading-Strand DNA Polymerase into the Eukaryotic Replisome. Curr. Biol. 23, 543-552. doi:10.1016/j.cub.2013.02.011

Sharma, N. M., Kochenova, O. V., and Shcherbakova, P. V. (2011). The Noncanonical Protein Binding Site at the Monomer-Monomer Interface of Yeast Proliferating Cell Nuclear Antigen (PCNA) Regulates the Rev1-PCNA Interaction and Pol//Rev1-dependent Translesion DNA Synthesis. J. Biol. Chem. 286, 33557-33566. doi:10.1074/jbc.M110.206680

Simon, A. C., Zhou, J. C., Perera, R. L., van Deursen, F., Evrin, C., Ivanova, M. E., et al. (2014). A Ctf4 Trimer Couples the CMG Helicase to DNA Polymerase $\alpha$ in the Eukaryotic Replisome. Nature 510, 293-297. doi:10.1038/nature13234

Sogo, J. M., Lopes, M., and Foiani, M. (2002). Fork Reversal and ssDNA Accumulation at Stalled Replication Forks Owing to Checkpoint Defects. Science 297, 599-602. doi:10.1126/science.1074023

Somyajit, K., Gupta, R., Sedlackova, H., Neelsen, K. J., Ochs, F., Rask, M.-B., et al. (2017). Redox-sensitive Alteration of Replisome Architecture Safeguards Genome Integrity. Science 358, 797-802. doi:10.1126/ science.aao3172

Sparks, J. L., Chistol, G., Gao, A. O., Räschle, M., Larsen, N. B., Mann, M., et al. (2019). The CMG Helicase Bypasses DNA-Protein Cross-Links to Facilitate Their Repair. Cell 176, 167-181.e21. doi:10.1016/j.cell.2018.10.053

Stelter, P., and Ulrich, H. D. (2003). Control of Spontaneous and Damage-Induced Mutagenesis by SUMO and Ubiquitin Conjugation. Nature 425, 188-191. doi:10.1038/nature01965

Stingele, J., Bellelli, R., and Boulton, S. J. (2017). Mechanisms of DNA-Protein Crosslink Repair. Nat. Rev. Mol. Cel Biol. 18, 563-573. doi:10.1038/nrm.2017.56

Sun, J., Shi, Y., Georgescu, R. E., Yuan, Z., Chait, B. T., Li, H., et al. (2015). The Architecture of a Eukaryotic Replisome. Nat. Struct. Mol. Biol. 22, 976-982. doi: $10.1038 / \mathrm{nsmb} .3113$

Šviković, S., Crisp, A., Tan-Wong, S. M., Guilliam, T. A., Doherty, A. J., Proudfoot, N. J., et al. (2019). R-loop Formation during S Phase Is Restricted by PrimPol-Mediated Repriming. EMBO J. 38, e99793. doi:10.15252/embj.201899793 
Taglialatela, A., Alvarez, S., Leuzzi, G., Sannino, V., Ranjha, L., Huang, J.-W., et al. (2017). Restoration of Replication Fork Stability in BRCA1- and BRCA2Deficient Cells by Inactivation of SNF2-Family Fork Remodelers. Mol. Cel 68, 414-430.e8. doi:10.1016/j.molcel.2017.09.036

Taylor, M. R. G., and Yeeles, J. T. P. (2018). The Initial Response of a Eukaryotic Replisome to DNA Damage. Mol. Cel 70, 1067-1080.e12. doi:10.1016/ j.molcel.2018.04.022

Taylor, M. R. G., and Yeeles, J. T. P. (2019). Dynamics of Replication Fork Progression Following Helicase-Polymerase Uncoupling in Eukaryotes. J. Mol. Biol. 431, 2040-2049. doi:10.1016/j.jmb.2019.03.011

Thangavel, S., Berti, M., Levikova, M., Pinto, C., Gomathinayagam, S., Vujanovic, M., et al. (2015). DNA2 Drives Processing and Restart of Reversed Replication forks in Human Cells. J. Cel Biol. 208, 545-562. doi:10.1083/jcb.201406100

Thomsen, N. D., and Berger, J. M. (2009). Running in Reverse: The Structural Basis for Translocation Polarity in Hexameric Helicases. Cell 139, 523-534. doi:10.1016/j.cell.2009.08.043

Tissier, A., Janel-Bintz, R., Coulon, S., Klaile, E., Kannouche, P., Fuchs, R. P., et al. (2010). Crosstalk between Replicative and Translesional DNA Polymerases: PDIP38 Interacts Directly with Poln. DNA Repair 9, 922-928. doi:10.1016/ j.dnarep.2010.04.010

Toledo, L. I., Altmeyer, M., Rask, M.-B., Lukas, C., Larsen, D. H., Povlsen, L. K., et al. (2013). ATR Prohibits Replication Catastrophe by Preventing Global Exhaustion of RPA. Cell 155, 1088-1103. doi:10.1016/j.cell.2013.10.043

Toledo, L., Neelsen, K. J., and Lukas, J. (2017). Replication Catastrophe: When a Checkpoint Fails Because of Exhaustion. Mol. Cel 66, 735-749. doi:10.1016/ j.molcel.2017.05.001

Tsuda, M., Ogawa, S., Ooka, M., Kobayashi, K., Hirota, K., Wakasugi, M., et al. (2019). PDIP38/PolDIP2 Controls the DNA Damage Tolerance Pathways by Increasing the Relative Usage of Translesion DNA Synthesis over Template Switching. PLOS ONE 14, e0213383. doi:10.1371/journal.pone.0213383

Unk, I., Hajdú, I., Fátyol, K., Szakál, B., Blastyák, A., Bermudez, V., et al. (2006). Human SHPRH Is a Ubiquitin Ligase for Mms2-Ubc13-dependent Polyubiquitylation of Proliferating Cell Nuclear Antigen. Proc. Natl. Acad. Sci. 103, 18107-18112. doi:10.1073/pnas.0608595103

Vaisman, A., and Woodgate, R. (2017). Translesion DNA Polymerases in Eukaryotes: what Makes Them Tick? Crit. Rev. Biochem. Mol. Biol. 52, 274-303. doi:10.1080/10409238.2017.1291576

Villa, F., Simon, A. C., Ortiz Bazan, M. A., Kilkenny, M. L., Wirthensohn, D., Wightman, M., et al. (2016). Ctf4 Is a Hub in the Eukaryotic Replisome that Links Multiple CIP-Box Proteins to the CMG Helicase. Mol. Cel 63, 385-396. doi:10.1016/j.molcel.2016.06.009

Vrtis, K. B., Dewar, J. M., Chistol, G., Wu, R. A., Graham, T. G. W., and Walter, J. C. (2021). Single-strand DNA Breaks Cause Replisome Disassembly. Mol. Cel 81, 1309-1318. doi:10.1016/j.molcel.2020.12.039

Wan, L., Lou, J., Xia, Y., Su, B., Liu, T., Cui, J., et al. (2013). hPrimpol1/ CCDC111 Is a Human DNA Primase-polymerase Required for the Maintenance of Genome Integrity. EMBO Rep. 14, 1104-1112. doi:10.1038/embor.2013.159

Wang, Z., and Xiao, W. (2020). Distinct Requirements for Budding Yeast Rev1 and Poln in Translesion DNA Synthesis across Different Types of DNA Damage. Curr. Genet. 66, 1019-1028. doi:10.1007/s00294-020-01092-w

Wasserman, M. R., Schauer, G. D., O’Donnell, M. E., and Liu, S. (2019). Replication Fork Activation Is Enabled by a Single-Stranded DNA Gate in CMG Helicase. Cell 178, 600-611.e16. doi:10.1016/j.cell.2019.06.032

Watanabe, K., Tateishi, S., Kawasuji, M., Tsurimoto, T., Inoue, H., and Yamaizumi, M. (2004). Rad18 Guides Poln to Replication Stalling Sites through Physical Interaction and PCNA Monoubiquitination. EMBO J. 23, 3886-3896. doi:10.1038/sj.emboj.7600383

Wilson, R. C., Jackson, M. A., and Pata, J. D. (2013). Y-family Polymerase Conformation Is a Major Determinant of Fidelity and Translesion Specificity. Structure 21, 20-31. doi:10.1016/j.str.2012.11.005

Wu, Y., Shin-ya, K., and Brosh, R. M. (2008). FANCJ Helicase Defective in Fanconia Anemia and Breast Cancer Unwinds G-Quadruplex DNA to Defend Genomic Stability. Mol. Cel. Biol. 28, 4116-4128. doi:10.1128/MCB.02210-07
Wu, W., Rokutanda, N., Takeuchi, J., Lai, Y., Maruyama, R., Togashi, Y., et al. (2018). HERC2 Facilitates BLM and WRN Helicase Complex Interaction with RPA to Suppress G-Quadruplex DNA. Cancer Res. 78, 6371-6385. doi:10.1158/ 0008-5472.CAN-18-1877

Wu, R. A., Semlow, D. R., Kamimae-Lanning, A. N., Kochenova, O. V., Chistol, G., Hodskinson, M. R., et al. (2019). TRAIP Is a Master Regulator of DNA Interstrand Crosslink Repair. Nature 567, 267-272. doi:10.1038/s41586-019-1002-0

Wu, R. A., Pellman, D. S., and Walter, J. C. (2021). The Ubiquitin Ligase TRAIP: Double-Edged Sword at the Replisome. Trends Cel Biol. 31, 75-85. doi:10.1016/ j.tcb.2020.11.007

Yang, W., and Gao, Y. (2018). Translesion and Repair DNA Polymerases: Diverse Structure and Mechanism. Annu. Rev. Biochem. 87, 239-261. doi:10.1146/ annurev-biochem-062917-012405

Yeeles, J. T. P., Poli, J., Marians, K. J., and Pasero, P. (2013). Rescuing Stalled or Damaged Replication Forks. Cold Spring Harb. Perspect. Biol. 5, a012815. doi:10.1101/cshperspect.a012815

Yeeles, J. T. P., Deegan, T. D., Janska, A., Early, A., and Diffley, J. F. X. (2015). Regulated Eukaryotic DNA Replication Origin Firing with Purified Proteins. Nature 519, 431-435. doi:10.1038/nature14285

Yeeles, J. T. P., Janska, A., Early, A., and Diffley, J. F. X. (2017). How the Eukaryotic Replisome Achieves Rapid and Efficient DNA Replication. Mol. Cel 65, 105-116. doi:10.1016/j.molcel.2016.11.017

Yuan, Z., Bai, L., Sun, J., Georgescu, R., Liu, J., O’Donnell, M. E., et al. (2016). Structure of the Eukaryotic Replicative CMG Helicase Suggests a Pumpjack Motion for Translocation. Nat. Struct. Mol. Biol. 23, 217-224. doi:10.1038/nsmb.3170

Yuan, Z., Georgescu, R., Santos, R. d. L. A., Zhang, D., Bai, L., Yao, N. Y., et al. (2019). Ctf4 Organizes Sister Replisomes and Pol a into a Replication Factory. eLife 8, e47405. doi:10.7554/eLife.47405

Yuan, Z., Georgescu, R., Bai, L., Zhang, D., Li, H., and O'Donnell, M. E. (2020a). DNA Unwinding Mechanism of a Eukaryotic Replicative CMG Helicase. Nat. Commun. 11, 688. doi:10.1038/s41467-020-14577-6

Yuan, Z., Georgescu, R., Schauer, G. D., O’Donnell, M. E., and Li, H. (2020b). Structure of the Polymerase $\varepsilon$ Holoenzyme and Atomic Model of the Leading Strand Replisome. Nat. Commun. 11, 3156. doi:10.1038/s41467-020-16910-5

Zellweger, R., Dalcher, D., Mutreja, K., Berti, M., Schmid, J. A., Herrador, R., et al. (2015). Rad51-mediated Replication fork Reversal Is a Global Response to Genotoxic Treatments in Human Cells. J. Cel Biol. 208, 563-579. doi:10.1083/ jcb.201406099

Zeman, M. K., and Cimprich, K. A. (2014). Causes and Consequences of Replication Stress. Nat. Cel Biol. 16, 2-9. doi:10.1038/ncb2897

Zhang, J., Dewar, J. M., Budzowska, M., Motnenko, A., Cohn, M. A., and Walter, J. C. (2015). DNA Interstrand Cross-Link Repair Requires Replication-fork Convergence. Nat. Struct. Mol. Biol. 22, 242-247. doi:10.1038/nsmb.2956

Zhao, L., and Washington, M. (2017). Translesion Synthesis: Insights into the Selection and Switching of DNA Polymerases. Genes 8, 24. doi:10.3390/ genes8010024

Zheng, F., Georgescu, R. E., Li, H., and O'Donnell, M. E. (2020). Structure of Eukaryotic DNA Polymerase $\delta$ Bound to the PCNA Clamp while Encircling DNA. Proc. Natl. Acad. Sci. USA 117, 30344-30353. doi:10.1073/pnas.2017637117

Zhou, J. C., Janska, A., Goswami, P., Renault, L., Abid Ali, F., Kotecha, A., et al. (2017). CMG-Pol Epsilon Dynamics Suggests a Mechanism for the Establishment of Leading-Strand Synthesis in the Eukaryotic Replisome. Proc. Natl. Acad. Sci. USA 114, 4141-4146. doi:10.1073/pnas.1700530114

Conflict of Interest: The author declares that the research was conducted in the absence of any commercial or financial relationships that could be construed as a potential conflict of interest.

Copyright (C) 2021 Guilliam. This is an open-access article distributed under the terms of the Creative Commons Attribution License (CC BY). The use, distribution or reproduction in other forums is permitted, provided the original author(s) and the copyright owner(s) are credited and that the original publication in this journal is cited, in accordance with accepted academic practice. No use, distribution or reproduction is permitted which does not comply with these terms. 